\section{The Myogenesis Program Drives Clonal Selection and Drug Resistance in Rhabdomyosarcoma}

Departments of ${ }^{1}$ Developmental Neurobiology, ${ }^{2}$ Oncology, ${ }^{3}$ Computational Biology, ${ }^{6}$ Radiation Oncology and ${ }^{7}$ Pathology at St. Jude Children's Research Hospital, Memphis, Tennessee 38105, USA

${ }^{4}$ Department of Pathology, University of Colorado School of Medicine, Aurora, Colorado 80045, USA

${ }^{5}$ Department of Mathematics, University of California, Irvine, California 92697, USA

${ }^{8}$ Department of Population Health and Disease Prevention, Program in Public Health, Susan and Henry Samueli College of Health Sciences, University of California, Irvine, California 92697 USA

*These authors contributed equally

Correspondence and requests for materials should be addressed to:

Michael A. Dyer

Department of Developmental Neurobiology, MS-323

St. Jude Children's Research Hospital

262 Danny Thomas Place, Memphis, TN 38105-3678, USA

Phone: (901) 595-2257

Fax: (901) 595-3143

E-mail: michael.dyer@stjude.org 


\section{Abstract}

2 Rhabdomyosarcoma (RMS) is a pediatric cancer with features of skeletal muscle; patients with

3 unresectable or metastatic RMS fare poorly due to high rates of disease recurrence. Here, we use

$4 \quad$ single cell and single nucleus RNA-sequencing to show that RMS tumors recapitulate the

5 spectrum of embryonal myogenesis. Using matched patient samples from a clinical trial and

6 orthotopic patient-derived xenografts (O-PDXs), we show chemotherapy eliminates the most

7 proliferative component with features of myoblasts; after treatment, the quiescent immature

8 population with features of paraxial mesoderm expands to reconstitute the developmental

9 hierarchy of the original tumor. We discovered that this paraxial mesoderm population is

10 dependent on EGFR signaling and is sensitive to EGFR inhibitors. Taken together, this data

11 serves as a proof-of-concept that targeting each developmental state in RMS is an effective

12 strategy for improving outcomes by preventing disease recurrence. 


\section{Introduction}

2 Rhabdomyosarcoma (RMS) is the most common pediatric soft tissue sarcoma and has molecular,

3 cellular and histopathologic features of developing skeletal muscle ${ }^{1-3}$. The alveolar form of RMS

4 (ARMS) is more differentiated than the embryonal form (ERMS) and each subtype has distinct

5 genomic and epigenomic landscapes ${ }^{2,4,5}$. For newly diagnosed RMS patients, the overall survival

6 rate is $70 \%$ using multiagent chemotherapy combined with radiation and/or surgical resection ${ }^{6,7}$.

7 Unfortunately, a subset of patients experience disease recurrence after treatment completion; for

8 those patients, overall survival rate drops below $20 \%{ }^{8}$. Genomic studies have shown that clonal

9 selection occurs with disease recurrence, but no recurrent genetic lesion has been identified that

10 contributes to survival of the rare clones of cells for $\mathrm{RMS}^{2,5,9}$. This raises the possibility that

11 other, non-genetic mechanisms may contribute to drug resistance and disease recurrence in RMS.

12 To explore this possibility, we performed single cell (sc) and single nucleus (sn) RNA-seq of

13 RMS patient tumors and matched orthotopic patient-derived xenografts (O-PDXs). We also

14 performed lentiviral barcode labeling to trace the clonal expansion of individual tumor cells

15 during normal growth and in response to treatment. Taken together, these studies showed that

16 individual tumor cells transition through myogenesis and the underlying myogenic

17 developmental hierarchy contributes to clonal selection with treatment. We used the

18 developmental program in RMS to identify therapeutic vulnerabilities that could be exploited to

19 reduce disease recurrence. Overall, this study reveals a developmental hierarchy with RMS and

20 introduces a novel approach to treating pediatric cancers, wherein targeting specific

21 developmental states that are destined to persist during therapy can be used to improve treatment

22 efficacy. 


\section{Results}

\section{RMS tumors have developmental heterogeneity}

3 Skeletal muscle develops from the mesodermal cells of the somites during embryogenesis and

4 undergoes stepwise differentiation, which is typified by the expression of myogenic regulatory

5 factors $^{10,11}$ (MRFs; Fig. 1A,B). RMS tumors have features of skeletal muscle including

6 myofibers and heterogenous expression of proteins such as myogenin (MYOG) ${ }^{3,12}$. To further

7 investigate the transcriptomic heterogeneity within RMS, we performed droplet-based single-cell

8 RNA-sequencing (scRNA-seq) (Extended Data Tables 1 and 2). We obtained fresh ERMS and

9 ARMS patient tumor tissue (Fig. 1C,D) following surgical resection and generated single-cell

10 suspensions (>90\% viable cells) for 3'-directed scRNA-seq (10x Genomics). Inference of

11 somatic copy number alterations was used to distinguish malignant cells from non-malignant

12 cells $^{13,14}$ (Extended Data Fig. 1).

Single-cell analysis showed there were distinct populations of cells expressing

14 transcription factors characteristic of paraxial mesoderm (MEOX2, PAX3), myoblasts (MYF5,

$15 M S C$ ) and myocytes (MYOG, MEF2C) (Fig. 1E,F and data not shown). The proportion of $M Y O G$

16 expressing cells in the scRNA-seq data was consistent with the proportion measured by

17 immunohistochemical staining (IHC) (Fig. 1C-F). The ARMS sample had fewer tumor cells

18 expressing the early paraxial mesoderm MRF MEOX2 (2.1\%) than the ERMS sample (29.4\%),

19 and more cells expressing the late myocyte MRF MYOG (75.2\% versus 25.6\%; Fig. 1E,F). RNA

20 velocity analysis, which leverages the simultaneous measurement of spliced and unspliced RNA

21 transcripts to generate a model of the future state of cells ${ }^{15}$, showed unidirectional transit of cells

22 from the paraxial mesoderm through myoblast to the myocyte state in the ERMS tumor (Fig. 
1G,H). Non-malignant cells including monocytes, fibroblasts, lymphocytes and vascular

2

endothelial cells were readily identifiable in our scRNA-seq dataset (Extended Data Fig. 1).

The rarity of childhood cancers limits the ability to obtain fresh tissue samples for scRNA-seq. To increase the number of evaluable tumors, we validated single-nucleus RNAsequencing (snRNA-seq) of frozen tumor tissue and adapted our computational pipeline to accommodate data generated from snRNA-seq ${ }^{14}$. Specifically, we compared scRNA-seq from fresh tumors (SJRHB030680_R1 and SJRHB031320_D1) (Fig. 1E,F) to snRNA-seq of matched frozen tumor specimens (Extended Data Fig. 2). As shown previously for neuroblastoma ${ }^{14}$, we were able to recover more cells of the tumor microenvironment (TME) by snRNA-seq compared to data generated by scRNA-seq (Extended Data Fig. 2). To extend our single cell transcriptional profiling, we performed snRNA-seq on 18 RMS tumors (12 ERMS and 6 ARMS) (Extended Data Tables 1 and 2). In total, 122,731 nuclei were analyzed from the patient tumors. As for the fresh tumors, copy number inference was used to distinguish malignant nuclei $(111,474)$ from the normal nuclei $(11,257)$ in the TME. The malignant nuclei were integrated using Conos, an approach that leverages inter-sample mappings to generate a unified graph for the identification of communal cell clusters ${ }^{16}$ (Fig. 2A). Leiden clustering identified 7 clusters, which we combined into 1 mesoderm, 4 myoblast and 2 myocyte signature groups based on expression of MRFs (Fig. 2B). The 2 myocyte populations were distinguished by expression of genes involved in muscle differentiation and function (Extended Data Table 3). The 4 myoblast populations were distinguished by ribosomal genes $\left(\mathrm{p}=4.3 \times 10^{-40}\right)$ and muscle differentiation genes $(\mathrm{p}=0.0005)($ Extended Data Table 3). We identified 954 differentially expressed genes, of which 945 were cluster-type specific (Extended Data Table 3). Extracellular matrix and cell adhesion pathways were enriched in the paraxial mesoderm-like tumor cells, ribosome biosynthesis 
1 pathways were enriched in the myoblast-like cells and pathways involved in muscle function

2 were enriched in the myocyte-like cells (Extended Data Table 3). While all the tumors had a

3 mixture of cells with mesoderm, myoblast, and myocyte signatures, ARMS tumors contained

4 significantly fewer cells with the mesodermal gene expression signature $(\mathrm{p}=0.008$; unpaired $\mathrm{t}$ -

5 test) and were skewed towards the myocyte signature (Fig. 2C and Extended Data Fig. 3). One

6 ERMS tumor (SJRHB010928_R1) was notable in that it contained a majority (97\%) of tumor

7 cells with the mesodermal signature (Extended Data Fig. 3A,B). This sample was collected

8 during treatment (Extended Data Table 1) suggesting that mesodermal cells are more resistant to

9 treatment than the other cell populations. The proliferating cells were significantly enriched in

10 the myoblast population ( $\mathrm{p}<0.0001$; one-way ANOVA with multiple comparisons) (Fig. 2E,F).

11 All data can be viewed using an interactive viewer at: https://pecan.stjude.cloud/static/RMS-

12 scrna-atlas-2020/.

The same approach was used to cluster the non-malignant cells within the TME

14 (Extended Data Figure 3C-F). Comparing normal cell populations between ERMS and ARMS

15 showed that fibroblasts in ARMS were significantly enriched in pathways involved in

16 extracellular matrix synthesis and organization as well as cell adhesion. In addition, SFRP2 and

17 SFRP4 were significantly $\left(\mathrm{p}<1 \times 10^{-90}\right)$ enriched in fibroblasts from ARMS (45\% and $56 \%$ of

18 cells, respectively) relative to ERMS (1\% and 3\%, respectively) (Extended Data Table 4). A

19 previous pan-cancer analysis showed that $S F R P 2$ and SFRP4 represent a tightly regulated

20 transcriptional program in cancer stroma that correlates with poor prognosis, EMT and

21 angiogenesis across multiple cancers ${ }^{17}$. The $H L A-A, B, C, E$ and $B 2 M$ and $C D 74$ genes were

22 significantly upregulated in lymphocytes from ARMS and $H L A-D R A, D R B 1$ and $D P B 1$ were

23 significantly upregulated in monocytes from ERMS (Extended Data Table 4). 
We next investigated the spatial heterogeneity of malignant subpopulations using single and multiplex immunohistochemistry (IHC) on 12 patient tumor specimens. Consistent with our transcriptomic findings, there was heterogenous expression of MEOX2, MYF5 and MYOG

4 protein (Fig. 2G). The proportion of immunopositive cells were correlated with the proportion of each population from the sc/snRNA-seq (Fig. 2H). Double IHC showed that these proteins were expressed in a mutually exclusive pattern consistent with the distinct clusters of mesoderm, myoblast and myocyte populations in RMS tumors from sc/snRNA-seq (Fig. 2I,J and data not

8 shown).

\section{Developmental indexing of RMS using embryonic snRNA-seq data}

11 To extend our analysis of the developmental trajectory of RMS beyond MRFs, we analyzed our RMS data within the context of early muscle development using a single-nucleus atlas of organogenesis from mouse embryos at E9.5, E10.5, E11.5, E12.5, and E13.5 ${ }^{18}$. We extracted data from the skeletal muscle lineage and performed trajectory analysis on half of the data to generate a training dataset (Fig. 3A-D). We then adapted latent cellular analysis (LCA) ${ }^{19}$ to

16 calculate the similarity in the latent cellular space between cells in the remaining half of the 17 skeletal muscle dataset to cells used for training; a normalized muscle developmental index was

18 then calculated for each nucleus within the validation dataset (Fig. 3E,F). The developmental 19 index increased with embryonic age as expected within the validation dataset (Fig. 3E,F). Using this unsupervised developmental indexing approach, we confirmed that individual

21 RMS tumors have cellular heterogeneity that reflects normal myogenesis. For example, in

22 SJRHB030680_R1, an ERMS tumor, we identified a broad range of developmental indices

23 within the malignant components of the tumor (Fig. 3G). In contrast, in SJRHB031320_D1, an 
1 ARMS tumor, the range of developmental indices was narrower and more skewed toward later

2 stages of myogenesis (Fig. 3H). Using our entire patient cohort of 18 tumors, we were able to

3 generalize these findings to RMS tumors - ERMS tumors had a wide diversity of developmental

4 indices while ARMS tumors narrowly centered with developmental indices from later stages of

5 murine myogenesis (Fig. 3I).

6

7 O-PDXs and organoids recapitulate clonal heterogeneity in RMS

8 We have previously established a panel of RMS O-PDXs and shared those models through the

9 Childhood Solid Tumor Network ${ }^{9}$. These O-PDXs encompass the clinical and molecular

10 diversity of RMS, and have previously undergone bulk genomic, transcriptomic, proteomic and

11 epigenomic analyses ${ }^{2,4,9}$. We expanded our single-cell transcriptomic profiling to include the O-

12 PDXs that correspond to the 18 patient tumors profiled here (Extended Data Table 2 and

13 https://pecan.stjude.cloud/static/RMS-scrna-atlas-2020/). We performed the same analyses,

14 including developmental indexing (Fig. 3J). All 3 cell types (mesoderm, myoblast, and myocyte)

15 were preserved in the O-PDXs in the snRNA-seq and IHC analysis (Extended Data Fig. 4 and

16 data not shown). As expected, the O-PDXs lacked normal cells from the patient TME but

17 contained infiltration of murine monocytes (Extended Data Fig. 4). The patient tumor that was

18 collected during treatment and was enriched in cells with the mesodermal signature

19 (SJRHB010928_R1) re-established the developmental hierarchy in the O-PDX

20 (SJRHB010928_X1) (Extended Data Fig. 4).

21 To complement the O-PDXs, we also evaluated the transcriptomic heterogeneity of ex

22 vivo organoids derived from the O-PDXs (Supplemental Methods). Malignant cells within

23 organoids shared the cellular diversity seen in the originating patient tumor and O-PDX by single 
1 cell transcriptional profiling (Extended Data Fig. 4). IHC for MEOX2, MYF5 and MYOG for

2 the organoids showed a similarity to their matched patient tumor and corresponding O-PDX

3 (Extended Data Fig. 4 and data not shown).

4

\section{$5 \quad$ RMS cells transition through developmental states}

6 RNA velocity analysis (see Fig. 1G,H) suggests that individual RMS tumor cells may transition

7 through developmental stages from mesoderm to myoblast and myocyte (Fig. 4A). Alternatively,

8 it is possible that there are distinct clones of cells that are restricted to their developmental stage

9 (Fig. 4B). To distinguish between these two possibilities, we used a lentiviral barcoding library

10 that incorporates a unique oligonucleotide barcode into the 3'-untranslated region of blue

11 fluorescent protein (BFP) $)^{20,21}$ (Fig. 4C,D). We infected 15 of the O-PDX models with the

12 barcode library and analyzed the barcode distribution in vivo by scRNA-seq. Following scRNA-

13 seq library generation, the barcode is retrievable by a separate PCR amplification step. In each of

14 the tumors that we analyzed, individual barcodes were found across all tumor cell types

15 (mesoderm, myoblast and myocytes) (Fig. 4E-G and Extended Data Table 5). Taken together,

16 these lineage tracing data, RNA-velocity analyses and genetic clonal analysis are consistent with

17 a model in which individual ERMS tumor cells can transition through developmental stages. The

18 same was true for ARMS tumors but the population of cells with paraxial mesoderm gene

19 expression signature was lower so some barcodes were found only in the myoblast and myocyte

20 population (Extended Data Table 5).

21

22 Tumor cell heterogeneity reflects differential enhancer activity 
1 Several of the MRF genes that are turned on and off as cells transition through developmental

2 stages have core regulatory circuit super-enhancers ${ }^{4}$ (CRC-SEs) (Fig. 4H and Extended Data

3 Table 6). For example, MEOX2 and NFIX (mesoderm), PAX7 and CREB5 (myoblast) and

4 FOXO1 and SOX6 (myocyte) each have CRC-SEs (Extended Data Table 6). To determine if the

5 chromatin accessibility of those CRC-SEs changes as individual cells transition through the

6 myogenic differentiation program, we performed droplet-based single-cell assay of transposase-

7 accessible chromatin sequencing (scATAC-seq) on 7 O-PDX tumors. We integrated scATAC-

8 seq and scRNA-seq profiles to investigate the chromatin accessibility of CRC-SEs for MRFs in

9 developmentally distinct subpopulations (Fig. 4H-J and Extended Data Table 6). Transferring

10 cell labels between scRNA-seq data and scATAC-seq data in SJRHB010927_X1 enabled us to

11 identify cell-type specific enhancer regions in $M Y O D 1, M S C, M E O X 2$ and several other

12 myogenic genes (Fig. 4H-J and Extended Data Table 6). These regions correspond to previously

13 reported core regulatory circuit domains for those genes ${ }^{4,22}$. Analysis of all 7 O-PDX tumors

14 showed CRC-SEs that change in their chromatin accessibility in tumor cells with mesoderm

15 (MEOX2, SMAD3), myoblast (CREB5, PAX7), and myocyte (MYOD1, FOXO1) features

16 (Extended Data Table 6 and Extended Data Fig. 5). Collectively, these scATAC-seq studies

17 indicate that heterogeneity of developmental states within RMS tumors is reflected in chromatin

18 dynamics for myogenic CRC-SEs and genes.

\section{The mesoderm-like RMS cells are drug resistant}

21 Current chemotherapeutic regimens for RMS include drugs that target proliferating cells. The

22 myoblast population has the highest proportion of dividing cells in the patient tumors, the O-

23 PDXs, and the ex vivo organoids (Fig. 5A,B and data not shown). In a pair of matched ERMS 
1 samples obtained before and during treatment, SJRHB000026_R2 and SJRHB000026_R3

2 (Extended Data Fig. 3A), we noted that the post-treatment sample was skewed towards

3 mesoderm signature-expressing cells (28.6\% post-treatment versus $3.4 \%$ pre-treatment) with a

4 concomitant reduction in cells expressing the myocyte signature (1.4\% post-treatment versus

$531.4 \%$ pre-treatment). Additionally, one ERMS patient tumor, SJRHB010928_R1, was obtained

6 during treatment with fewer than 5\% viable cells by histology; in this sample, the majority

7 (96.8\%) of remaining viable cells expressed the mesoderm signature (Extended Data Fig. 3).

8 Taken together, these data suggest that the myoblast population may be more sensitive to

9 chemotherapy and the mesoderm-like population is more likely to survive treatment. To

10 investigate this trend further, we evaluated matched formalin-fixed paraffin embedded (FFPE)

11 tissue from 11 patients obtained at diagnosis and mid-treatment on a single therapeutic clinical

12 trial, RMS13 (NCT01871766). We quantitated the number of cells in each sample expressing

13 MEOX2 and MYOG (Extended Data Table 7). There was a significant enrichment in MEOX2

14 immunopositive cells in the post-treatment tumors relative to the matched pre-treatment RMS

15 samples and a corresponding decrease in MYOG immunopositive cells (Fig. 5C).

16 To model clonal selection in the laboratory, we generated longitudinal samples from

17 repeat biopsy of O-PDXs treated with a standard drug combination used to treat patients with

18 RMS (vincristine (VCR) and irinotecan (IRN)) at clinically relevant doses and schedules ${ }^{4,9}$ (Fig.

19 5D). For each O-PDX (SJRHB000026_X1, SJRHB013758_X1, SJRHB011_X,

20 SJRHB013757_X1 and SJRHB013759_X14), biopsies were performed at multiple timepoints

21 (before treatment (day 0), day 3, day 7, day 14 and day 21 of the first course) when sufficient

22 tumor was present to sample (Fig. 5E,F). We also collected tumor biopsies after the tumors

23 recurred. A portion of each biopsy underwent formalin-fixation for IHC staining for MEOX2, 
1 MYF5 and MYOG (Fig. 5G,H and data not shown). The remaining biopsy portion was utilized

2 for quantitative RT-PCR for 21 genes expressed in mesoderm, myoblast and myocyte-like RMS

3 tumor cells or snRNA-seq. In total, 250 biopsies were collected and 6,480 qRT-PCR reactions

4 were performed (Extended Data Table 8-13). As in patient samples, the myoblast and myocyte

5 populations were sensitive to treatment and the mesoderm tumor cells population was enriched

6 (Fig. 5I-K and Extended Data Table 8-13). Moreover, the normally quiescent mesoderm-like

7 cells re-entered the cell cycle to initiate myogenesis (Fig. 5K).

Taken together, our data suggest that ERMS tumor cells transition through distinct states

9 that represent progressive stages of myogenesis. These different states (paraxial mesoderm, myoblast, myocyte) have distinct proliferation properties and differential sensitivity to

11 chemotherapy. To further refine our understanding of the cellular heterogeneity of ERMS

12 tumors, their developmental trajectory and clonal selection with treatment, we developed a

13 mathematical model that follows the fate of cells in both 3-dimensional space and time.

14 Experimentally determined barcode distribution in each compartment was used to develop the

15 model (Fig. 5L), and barcode diversity was tracked over time. We assumed that upon cell

16 division, cells maintain their barcodes and we included barcoded and non-barcoded cells to

17 reflect the in vivo experiments. The relative proportion of different division types (self-

18 renewing/differentiating) in the mesodermal compartment determines whether the tissue remains

19 in homeostasis and influences the degree of clonal diversity loss over time. To parameterize the

20 model, we used experimental data from 10 barcoded ERMS xenografts. The fraction of dividing

21 cells and distribution of cells across compartments was determined from the sc/snRNA-seq data.

22 Our ERMS model predicts a decrease in clonal diversity (as measured by barcode diversity) over

23 time and clonal selection with treatment for individual tumors (Fig. 5M-P). To test this 
1 experimentally, we performed scRNA-seq on a barcoded ERMS tumor (SJRHB000026_X1)

2 after initial labeling and a subsequent passage in mice with and without clinically relevant

3 chemotherapy (vincristine+irinotecan). As predicted by the three-compartment model, there was

4 a decrease in clonal diversity over time and clonal selection with treatment (Fig. 5O-Q).

5 Additional iterations of modeling and comparison to in vivo barcode distribution data are

6 consistent with differential cytotoxicity across the cellular populations (mesoderm, myoblast,

7 myocyte). In particular, a subset of the mesoderm-like cells are proliferating thereby making

8 them sensitive to chemotherapy. Based on our model, partial elimination of the mesoderm-like

9 population is required to account for the clonal selection we observe in vivo in mice and in

10 patients.

EGFR is a therapeutic vulnerability in paraxial mesoderm RMS cells

13 Having shown that the paraxial mesoderm RMS cells are more quiescent and drug resistant than

14 the myoblast population, we set out to identify therapeutic vulnerabilities unique to this

15 population using a systems biology algorithm, NetBID (data-driven Network-based Bayesian

16 Inference of Drivers) ${ }^{23,24}$. NetBID, which was originally developed for bulk -omics data, was

17 adapted to analyze snRNA-seq profiles of our panel of 18 RMS patient tumors. We first used the

18 SJARACNe algorithm ${ }^{25}$ to reverse engineer cell type-specific interactomes for each of the 5

19 major cell types from the integrated snRNA-seq profiles (Fig. 6A). With a focus on signaling

20 drivers, we used the cell type-specific interactomes of 2,543 genes/proteins and inferred their

21 network activities in each nucleus using the NetBID algorithm. We then performed differential

22 activity analysis to identify cell type-specific therapeutic vulnerabilities in the RMS tumor cells

23 with the mesodermal signature. EGFR was significantly activated in the mesoderm population 
1 compared to myoblasts $\left(\mathrm{p}=4.4 \times 10^{-135}\right)$ and myocytes $\left(\mathrm{p}=1.8 \times 10^{-174}\right)$ and the network was rewired

2 as cells transition through the developmental hierarchy (Fig. 6B). EGFR network activity was

3 also significantly higher in ERMS relative to ARMS ( $\left.\mathrm{p}=5.4 \times 10^{-36}\right)$ (Fig. 6C,D). These data are

4 consistent with previous integrated epigenetic/proteomic analyses for differential pathway

5 activity in ERMS and $\mathrm{ARMS}^{4}$. In addition, previous studies have shown heterogenous

6 expression of EGFR protein in FFPE samples of $\mathrm{RMS}^{26-28}$. To validate these data, we performed

7 IHC for EGFR alone and in combination with markers of each cell population. We used the 5B7

8 monoclonal antibody which has been previously shown to correlate with EGFR inhibitor

9 (EGFRi) responsiveness in lung cancer $^{29}$. There was co-localization of EGFR with MEOX2 in 2-

10 color IHC and EGFR was mutually exclusive with MYOG (Fig. 6E,F).

11 To determine if EGFR is a therapeutic vulnerability in RMS, we exposed 3D ERMS

12 organoids that contain all 3 cell populations (Extended Data Fig. 6) to two different EGFRi's

13 (gefitinib and afatinib) with increasing concentrations of SN-38, the active metabolite of

14 irinotecan (Supplemental Information). The EGFRi's alone had no effect on overall organoid

15 viability as measured with CellTiter-Glo 3D which is not surprising given the low percentage of

16 mesoderm-like cells in the organoids (Extended Data Fig. 6). However, when the proliferating

17 myoblast population was targeted with increasing concentrations of SN-38, the addition of

18 EGFRi's significantly reduced viability (Extended Data Fig. 6). In a representative ERMS O-

19 PDX (SJRHB013758_X1), there was a significant improvement in outcome when afatinib or

20 gefitinib was combined with IRN+VCR (Fig. 6H,I). For a second ERMS O-PDX with a low

21 percentage of mesoderm-like cells, SJRHB010927_X1, O-PDXs responded robustly to

22 IRN+VCR therapy alone, reinforcing the importance of including standard-of-care treatment in

23 preclinical testing (Extended Data Fig. 6). 


\section{Discussion}

2 In conclusion, we have discovered that RMS tumor cells can transition through different stages

3 of myogenesis from an immature quiescent paraxial mesoderm state through a highly

4 proliferative myoblast stage and into a more differentiated myocyte state. Not only do cells

5 undergo changes in gene expression during these developmental transitions but super-enhancer

6 chromatin accessibility is also dynamic. While proliferating cells can be identified in tumor cell

7 population in patient tumors and O-PDXs, the most proliferative cells are in the myoblast stage.

8 It is therefore not surprising that broad spectrum chemotherapeutic regimens that target rapidly

9 dividing cells efficiently reduce tumor volume by killing the myoblast-like RMS tumor cells.

10 The immature paraxial mesoderm-like RMS tumor cells are more quiescent and can survive

11 therapy and then expand to repopulate the myogenic lineage found in the primary tumor. These

12 observations are consistent with decades of clinical research showing that different combinations

13 of broad- spectrum chemotherapy or intensification of existing regimens have failed to improve

14 outcomes for children with $\mathrm{RMS}^{6,7}$. Those different treatment approaches are killing the rapidly

15 dividing myoblast-like cells and the resistant mesoderm-like cells survive and contribute to

16 disease recurrence. By focusing our investigation on the mesoderm-like cells, we identified a

17 dependence on EGFR that can be exploited with EGFRi in vivo. While there were only a small

18 number of mesoderm-like cells in ARMS tumors, we discovered a dramatic upregulation of

19 EGFR during treatment suggesting that EGFRi's may be useful for both types of

20 rhabdomyosarcoma. Our study shows that treatment for RMS and possibly other pediatric solid

21 tumors should focus on total clonal elimination rather than continuing to target just the most

22 proliferative cell population that makes up the bulk of the tumor. Such an approach may reduce

23 disease recurrence and improve survival and quality of life for children with solid tumors. 
1

2

\section{Figure Legends}

\section{Figure 1: Single-cell RNA-sequencing (scRNA-seq) reveal a developmental hierarchy}

within RMS. A-B, During fetal myogenesis, mesodermal cells of the somite migrate to form

skeletal muscle throughout the body (A). During that migration, these cells undergo stepwise differentiation typified by the transient expression of myogenic regulatory factors ${ }^{10}(\mathrm{~B})$. C-D, Photomicrographs of an embryonal RMS tumor, SJRHB030680_R1 (C) and an alveolar RMS tumor, SHRHB031320_D1. Left, H\&E staining. Right, Myogenin (MYOG) immunohistochemistry (IHC) with 20X magnification. inset, 80X magnification. E-F, UMAP visualization of 3,973 malignant cells from SJRHB030680_R1 (E) and 2,414 malignant cells from SJRHB031320_D1 (F). Cells are colored based on expression of MEOX2 (left), MYF5 (center), and MYOG (right). G-H, RNA velocity analysis of SJRHB030680_R1 (G) and SJRHB031320_D1 (H). Abbreviations: ERMS, embryonal rhabdomyosarcoma; UMAP, uniform manifold approximation and projection. Scale bars: C,D, $100 \mu \mathrm{m}$.

Figure 2: Identification of major cell clusters within patient RMS tumors using singlenucleus RNA-sequencing. A, LargeVis visualization of snRNA-seq of 111,474 nuclei from 18 integrated patient RMS tumors, colored based on sample. B, Heatmap showing expression of myogenic regulatory factor expression across seven Leiden clusters. C, Boxplot showing the percentage of malignant nuclei within each muscle developmental state for each tumor. The center line demarcates the median value with rectangle showing interquartile range (IQR) between the first and third quartiles. The vertical bars extending from the rectangles indicate maximum and minimum values with the exception of outliers that exceed more than 3 times the IQR. D-E, LargeVis visualization of Leiden clustering of snRNA-seq grouped based on 
expression of mesoderm, myoblast, or myocyte myogenic regulatory factors (D) or colored by

2 predicted cell cycle phase (E). F, Plot of the proportion of proliferating cells (S/G2/M phase) in

3 each group, estimated using gene signatures associated with $\mathrm{G} 1, \mathrm{~S}$, and $\mathrm{G} 2 / \mathrm{M}$ phases ${ }^{13}$. Circles

4 are ERMS and squares are ARMS. Center line and rectangle indicate the median and IQR as in

5 panel (C). Vertical bars indicate the maximum and minimum values with the exception of

6 outliers that exceed more than 3 times the IQR. G, Immunohistochemistry image of an ERMS

7 tumor, SJRHB013758_D2 stained with antibodies against MEOX2 (left), MYF5 (center) and

8 MYOG (right). H, Quantitation of the percentage of cells positive for MEOX2 (blue), MYF5

9 (green), or MYOG (red) immunohistochemical staining ( $x$ axis) compared to percentage of cells

10 within each developmental state as determined by snRNA-seq ( $y$ axis). I-J, Dual staining of

11 MEOX2 (purple) and MYOG (brown) within SJRHB013758 (I) with magnified view (J).

12 Abbreviations: ERMS, embryonal rhabdomyosarcoma; ARMS, alveolar rhabdomyosarcoma.

13 Scale bars: $\mathrm{G}, 10 \mu \mathrm{m}$.

15 Figure 3: Developmental indexing of patient RMS tumors and orthotopic patient-derived xenografts. A, UMAP plot of 1.5 million nuclei from the Mouse Organogenesis Cell Atlas ${ }^{18}$,

17 downsampled to 100,000 nuclei. Clusters are colored based on trajectory. B, UMAP plot of

18576,560 nuclei from the mesenchymal trajectory with identification of the skeletal myogenesis

19 sub-trajectory. Nuclei are colored based on Leiden cluster. C, UMAP plot of 58,573 nuclei of the

20 skeletal muscle sub-trajectory with computational clustering that identifies nuclei from early

21 mesodermal progenitors, paraxial mesoderm, myoblasts, myocytes and myotubes. D, Heatmap of

22 aggregated transcription from each cluster demonstrating expression of myogenic regulatory

23 factors and additional mesodermal markers. E, Violin plot of projected developmental indices of 
1 embryonic skeletal muscle data separated by mouse embryonic stage. F, UMAP plot of

2 developmental indices within the embryonic skeletal muscle sub-trajectory. G-H, Application of

3 developmental indices to an ERMS tumor, SJRHB030680_R1 (G) and an ARMS tumor,

4 SJRHB031320_D1 (H). I-J, Developmental indices of 18 patient RMS tumors (I) or 18 O-PDXs

5 (J). Abbreviations: ERMS, embryonal rhabdomyosarcoma; ARMS, alveolar rhabdomyosarcoma;

6 UMAP, uniform manifold approximation and projection.

Figure 4: Developmental status in ERMS is plastic and associated with chromatin

9 accessibility at core regulatory superenhancer regions. A-B, Two competing models of tumor heterogeneity within RMS. In the first model, RMS cells transition across developmental states

11 (A); in the alternate model, genetically distinct clones are restricted to muscle developmental

12 states (B). C, Schematic of the lentiviral barcode plasmid. ${ }^{20,21}$ An 18-mer of random nucleotides

13 is incorporated into the 3'-untranslated region of a blue fluorescent protein (BFP) tag, enabling

14 barcode recovery from scRNA-seq libraries. D, Plot of frequency of individual barcodes for

15 subsequent passages of an individual ERMS O-PDX, SJRHB00026_X1. E-F. UMAP plot of an

16 ERMS O-PDX SJRHB013758_X2, colored based on developmental stage (E), or with 3 specific

17 barcodes highlighted (F). G, Quantitation of the developmental state diversity of all tumor cells

18 within SJRHB013758_X2, and from the 5 most prevalent barcoded clones. H, ChIP-seq and

19 chromHMM of MYOD1 in an ERMS O-PDX, SJRHB10927_X1. Scales are indicated on the left,

20 and a previously identified CRC-SE ${ }^{4}$ is highlighted in blue. I, Comparison of H3K27

21 trimethylation in various pediatric O-PDXs. OS, osteosarcoma; EWS, Ewing sarcoma; LPS,

22 liposarcoma; HGS, high-grade sarcoma; NB, neuroblastoma. J, Single-cell ATAC-seq of

23 SJRHB010927_X1 at the MYOD1 locus; cell identities were defined via gene activity estimation, 
1 and dataset integration with scRNA-seq data ${ }^{30}$. Abbreviations: ERMS, embryonal

2 rhabdomyosarcoma; ARMS, alveolar rhabdomyosarcoma; UMAP, uniform manifold

3 approximation and projection; RMS, rhabdomyosarcoma; OS, osteosarcoma; EWS, Ewing

4 sarcoma; LPS, liposarcoma; HGS, high grade sarcoma; NB, neuroblastoma.

6 Figure 5. Chemotherapy treatment of ERMS selects for mesoderm developmental stages.

$7 \quad$ A-B, Bar plots showing percentage of cells predicted to be dividing within each developmental

8 stage for patient tumors (a) and O-PDXs (b). C, Plots showing immunopositivity for MEOX2

9 (left) and MYOG (right) in patient samples from RMS13 obtained before treatment ("diagnosis")

10 and during therapy ("mid-treatment"). D, Treatment schema for VI therapy of mice bearing RMS

11 O-PDXs. Needle biopsies were performed at days $0,3,7,14$, and 21 or when tumors were large

12 enough to sample. E, Photograph of needle biopsy of an orthotopically-injected xenograft. F-H,

13 Photograph of tissue obtained by a biopsied O-PDX (F), which was fixed and stained using H\&E

14 (G) or MYOG (H). I, Plot showing longitudinal expression of MEOX2 by qRT-PCR during

15 treatment. There is an increase in MEOX2 during chemotherapy (days 7,14,21) but the

16 proportion resets to basal levels after 28 days. This was verified by IHC (lower panel). J,

17 Boxplot of all biopsies for ERMS tumor bearing mice for the untreated and treated samples. The

18 plot is an integration of expression of 6 genes (MEOX2, PAX3, EGFR, CD44, DCN, POSTN)

19 expressed as normalized relative fold. The center line demarcates the median value with

20 rectangle showing interquartile range (IQR) between the first and third quartiles. The vertical

21 bars extending from the rectangles indicate maximum and minimum values with the exception of

22 outliers that more than 3 times the IQR. K, Relative proportion of nuclei in each developmental

23 state for longitudinal biopsies of a single O-PDX, determined using snRNA-seq of biopsied 
1 tissue. L, Diagram of the mathematical model of ERMS developmental heterogeneity. M-N,

2 Simulated average population size for an untreated ERMS tumor (M) or a treated ERMS tumor

3 (N) briefly exposed to an antiproliferative agent (gray bar). Average population size over 524

4 simulations are shown, standard error bars are too small to see. O-P, Simulated time course of

5 barcode dynamics for an ERMS tumor that was either untreated $(\mathrm{O})$ or briefly treated (P;

6 duration of treatment in grey bar). Each curve represents a different barcoded lineage. One

7 realization of the stochastic dynamics is shown. Insets under each graph show spatial

8 distributions of bar codes (color coded) in myoblast cells at an early and late stage of tumor

9 growth (O) and pre- and post-therapy (P). Q, Temporal development of the average entropy

10 index (measure of barcode diversity) during barcoded ERMS tumor growth, either untreated or

11 briefly treated (grey bar). Average entropy values over 524 simulations \pm standard errors (dashed

12 lines) are shown. Inset, bar plot comparing the initial entropy index to the final entropy index of

13 untreated or treated tumors in experiments. Model parameters were: average value of $\mathrm{L}_{\text {mes }}$

$14=0.0035\left(\mathrm{r}_{1}=1.5, \mathrm{r}_{2}=0.0001\right), \mathrm{L}_{\text {blast }}=0.0045, \mathrm{P}_{\mathrm{mes}}=0.55, \mathrm{P}_{\text {blast }}=0.49, \mathrm{D}=0.035, \alpha_{\mathrm{mes}}=0.0014, \alpha_{\text {blast }}$

$15=0.0035$. The parameter units are per minute. Abbreviations: ERMS, embryonal

16 rhabdomyosarcoma; ARMS, alveolar rhabdomyosarcoma; VCR, vincristine; IRN irinotecan.

Figure 6. Mesoderm-like ERMS cells are uniquely vulnerable to EGFR blockade. A,

19 Schematic workflow of NetBID algorithm to identify cell type-specific drivers from snRNA-seq

20 data. B, Volcano plot of differential activity analysis of signaling drivers in ERMSmesoderm vs.

21 other cell types. C-D, EGFR NetBID activity in different developmental states from snRNA-seq

22 data (C) and inferred from bulk RNA-seq of patient tumors (D). E-F, Dual IHC staining of

23 ERMS patient tumor, SJRHB030680_R1, combining EGFR (brown) with either MEOX2 (E) or 
1 MYOG (F) in purple. G, Schedules of drugs used for preclinical study. H, Survival curves for

2 each treatment group for a ERMS tumor O-PDX (SJRHB01378_X1). I, Representative image of

3 bioluminescence for mice treated on the study, scale bar is photons/sec/cm ${ }^{2}$ str. Scale bars: E,F,

$410 \mu \mathrm{m}$. Abbreviations: VCR, vincristine; IRN, irinotecan; ERMS, embryonal

5 rhabdomyosarcoma; ARMS, alveolar rhabdomyosarcoma. 
Materials and Methods

See Supplementary Information.

3

\section{Acknowledgements}

We thank A. McArthur for editing the manuscript. This work was supported by Cancer Center Support (CA21765) and grants to M.A.D. from the National Institutes of Health (EY014867, EY018599, and CA168875). The content is solely the responsibility of the authors and does not necessarily represent the official views of the National Institutes of Health. Research was also supported by American Lebanese Syrian Associated Charities. M.A.D. was also supported by the Howard Hughes Medical Institute, Alex’s Lemonade Stand, and Tully Family and Peterson Foundations. A.G.P. was supported by Alex's Lemonade Stand, Hyundai Hope on Wheels, and Damon Runyon Cancer Research Foundations.

\section{Author Contributions}

A.G.P, X.C. and M.A.D designed the study. A.G.P processed fresh and frozen tissue for singlecell/nucleus RNA-seq and ATAC-seq. B.G., K.B., and E.S. assisted in patient sample and xenograft tissue accrual for molecular analyses. A.G.P., X.C., X.H., and J.Y. performed computational analysis of data. M.R.C., B.A.O., and H.T. supervised immunohistochemical staining and pathology review. N.K. and D.W. performed mathematical modeling of tumors. M.J.K. and A.P. wrote and supervised the RMS13 clinical trial. A.K. manages the Childhood Solid Tumour Network. J.M. and K.B. assisted in molecular analysis. C.R. and X.Z. developed the single-cell viewer portal. K.B. and E.S. performed the preclinical testing and tumor biopsies. A.G.P. and J.L.N. developed the tumor organoid model. 


\section{Competing Interest Statement}

2 The authors declare no competing financial interests.

4 Supplementary Information

5 Supplementary Information is available for this paper.

7 Corresponding Author

8 Correspondence and requests for materials should be addressed to Michael Dyer at

9 michael.dyer@stjude.org.

11 Data and Source Code Availability

12 NetBID: https://jyyulab.github.io/NetBID

13 All processed single-cell and single-nucleus RNA-sequencing data are publicly accessible via an

14 online data portal (https://pecan.stjude.cloud/static/RMS-scrna-atlas-2020).

15 All raw sequencing data will be uploaded and be publicly available at the time of publication

16 through GEO accession GSE174376.

18 References

19 1. Ries, L. et al. Cancer Incidence and Survival among Children and Adolescents: United States SEER

20 Program 1975-1995. (National Cancer Institute, SEER Program).

21 2. Chen, X. et al. Targeting oxidative stress in embryonal rhabdomyosarcoma. Cancer Cell 24, 22 710-724 (2013).

23 3. Kahn, H. J. et al. Immunohistochemical and electron microscopic assessment of childhood 24 rhabdomyosarcoma. Increased frequency of diagnosis over routine histologic methods. Cancer 25 51, 1897-1903 (1983). 
4. Stewart, E. et al. Identification of Therapeutic Targets in Rhabdomyosarcoma through Integrated Genomic, Epigenomic, and Proteomic Analyses. Cancer Cell 34, 411-426 (2018).

5. Shern, J. F. et al. Comprehensive Genomic Analysis of Rhabdomyosarcoma Reveals a Landscape of Alterations Affecting a Common Genetic Axis in Fusion-Positive and FusionNegative Tumors. Cancer Discov 4, 216-231 (2014). Sarcomas. J Clin Oncol 36, 168-179 (2017).

8 7. Arndt, C. A. S., Bisogno, G. \& Koscielniak, E. Fifty Years of Rhabdomyosarcoma studies on

9 both sides of the Pond and Lessons Learned. Cancer Treat Rev 68, 94-101 (2018).

10 8. Pappo, A. S. et al. Survival After Relapse in Children and Adolescents With

11 Rhabdomyosarcoma: A Report From the Intergroup Rhabdomyosarcoma Study Group. J Clin

12 Oncol 17, 3487-3493 (1999).

13 9. Stewart, E. et al. Orthotopic patient-derived xenografts of paediatric solid tumours. Nature

14 549, 96-100 (2017).

15 10. Chal, J. \& Pourquié, O. Making muscle: skeletal myogenesis in vivo and in vitro.

16 Development 144, 2104-2122 (2017).

17 11. Buckingham, M. \& Rigby, P. W. J. Gene Regulatory Networks and Transcriptional

18 Mechanisms that Control Myogenesis. Dev Cell 28, 225-238 (2014).

12. Sebire, N. J. \& Malone, M. Myogenin and MyoD1 expression in paediatric rhabdomyosarcomas. J Clin Pathol 56, 412-416 (2003).

21 13. Tirosh, I. et al. Single-cell RNA-seq supports a developmental hierarchy in human

22 oligodendroglioma. Nature 539, 309-313 (2016).

23 14. Slyper, M. et al. A single-cell and single-nucleus RNA-Seq toolbox for fresh and frozen

24 human tumors. Nat Med 26, 792-802 (2020).

25 15. Manno, G. L. et al. RNA velocity of single cells. Nature 560, 494-498 (2018).

26 16. Barkas, N. et al. Joint analysis of heterogeneous single-cell RNA-seq dataset collections. Nat 27 Methods 16, 695-698 (2019).

28 17. Vincent, K. M. \& Postovit, L.-M. A pan-cancer analysis of secreted Frizzled-related proteins: 29 re-examining their proposed tumour suppressive function. Sci Rep 7, 42719 (2017).

30 18. Cao, J. et al. The single-cell transcriptional landscape of mammalian organogenesis. Nature

$31 \quad 566,496-502$ (2019). 
19. Cheng, C. et al. Latent cellular analysis robustly reveals subtle diversity in large-scale singlecell RNA-seq data. Nucleic Acids Res 16, e143 (2019).

20. Adamson, B. et al. A Multiplexed Single-Cell CRISPR Screening Platform Enables Systematic Dissection of the Unfolded Protein Response. Cell 167, 1867-1882 (2016).

5 21. Dixit, A. et al. Perturb-Seq: Dissecting Molecular Circuits with Scalable Single-Cell RNA 6 Profiling of Pooled Genetic Screens. Cell 167, 1853-1866 (2016).

23. Du, X. et al. Hippo/Mst signalling couples metabolic state and immune function of CD8 $\alpha+$ dendritic cells. Nature 558, 141-145 (2018).

11 24. Wijaya, J. et al. An ABC Transporter Drives Medulloblastoma Pathogenesis by Regulating

12 Sonic Hedgehog Signaling. Cancer Res 80, 1524-1537 (2020).

25. Khatamian, A., Paull, E. O., Califano, A. \& Yu, J. SJARACNe: a scalable software tool for gene network reverse engineering from big data. Bioinformatics 35, 2165-2166 (2018).

26. Ganti, R. et al. Expression and genomic status of EGFR and ErbB-2 in alveolar and embryonal rhabdomyosarcoma. Modern Pathol 19, 1213-1220 (2006).

27. Grass, B. et al. Immunohistochemical detection of EGFR, fibrillin-2, P-cadherin and AP2beta as biomarkers for rhabdomyosarcoma diagnostics. Histopathology 54, 873-879 (2009).

28. Wachtel, M. et al. Subtype and Prognostic Classification of Rhabdomyosarcoma by Immunohistochemistry. J Clin Oncol 24, 816-822 (2006).

29. Mascaux, C. et al. EGFR Protein Expression in Non-Small Cell Lung Cancer Predicts Response to an EGFR Tyrosine Kinase Inhibitor-A Novel Antibody for Immunohistochemistry or AQUA Technology. Clin Cancer Res 17, 7796-7807 (2011).

30. Stuart, T. et al. Comprehensive Integration of Single-Cell Data. Cell 177, 1888-1902 (2019).

31. Stewart, E. et al. The Childhood Solid Tumor Network: A new resource for the developmental biology and oncology research communities. Dev Biol 411, 287-293 (2016).

32. Smith, T., Heger, A. \& Sudbery, I. UMI-tools: modeling sequencing errors in Unique Molecular Identifiers to improve quantification accuracy. Genome Res 27, 491-499 (2017).

33. Butler, A., Hoffman, P., Smibert, P., Papalexi, E. \& Satija, R. Integrating single-cell transcriptomic data across different conditions, technologies, and species. Nat Biotechnol 36, $411-420$ (2018). 
1 34. Hafemeister, C. \& Satija, R. Normalization and variance stabilization of single-cell RNA-seq

2 data using regularized negative binomial regression. Genome Biol 20, 296 (2019).

3 35. Becht, E. et al. Dimensionality reduction for visualizing single-cell data using UMAP. Nat

4 Biotechnol 37, 38-44 (2019).

5 36. Wolf, F. A., Angerer, P. \& Theis, F. J. SCANPY: large-scale single-cell gene expression data 6 analysis. Genome Biol 19, 15 (2018).

7 37. Bergen, V., Lange, M., Peidli, S., Wolf, F. A. \& Theis, F. J. Generalizing RNA velocity to 8 transient cell states through dynamical modeling. Nat Biotechnol 1-7 (2020)

9 doi:10.1038/s41587-020-0591-3.

10 38. Aran, D. et al. Reference-based analysis of lung single-cell sequencing reveals a transitional 11 profibrotic macrophage. Nat Immunol 20, 163-172 (2019).

12 39. Traag, V. A., Waltman, L. \& Eck, N. J. van. From Louvain to Leiden: guaranteeing well13 connected communities. Sci Rep 9, 5233 (2019).

14 40. Saelens, W., Cannoodt, R., Todorov, H. \& Saeys, Y. A comparison of single-cell trajectory 15 inference methods. Nat Biotechnol 37, 547-554 (2019).

16 41. Street, K. et al. Slingshot: cell lineage and pseudotime inference for single-cell 17 transcriptomics. BMC Genomics 19, 477 (2018).

18 42. Hanawa, H. et al. Comparison of Various Envelope Proteins for Their Ability to Pseudotype 19 Lentiviral Vectors and Transduce Primitive Hematopoietic Cells from Human Blood. Mol Ther 20 5, 242-251 (2002). 
Figure 1

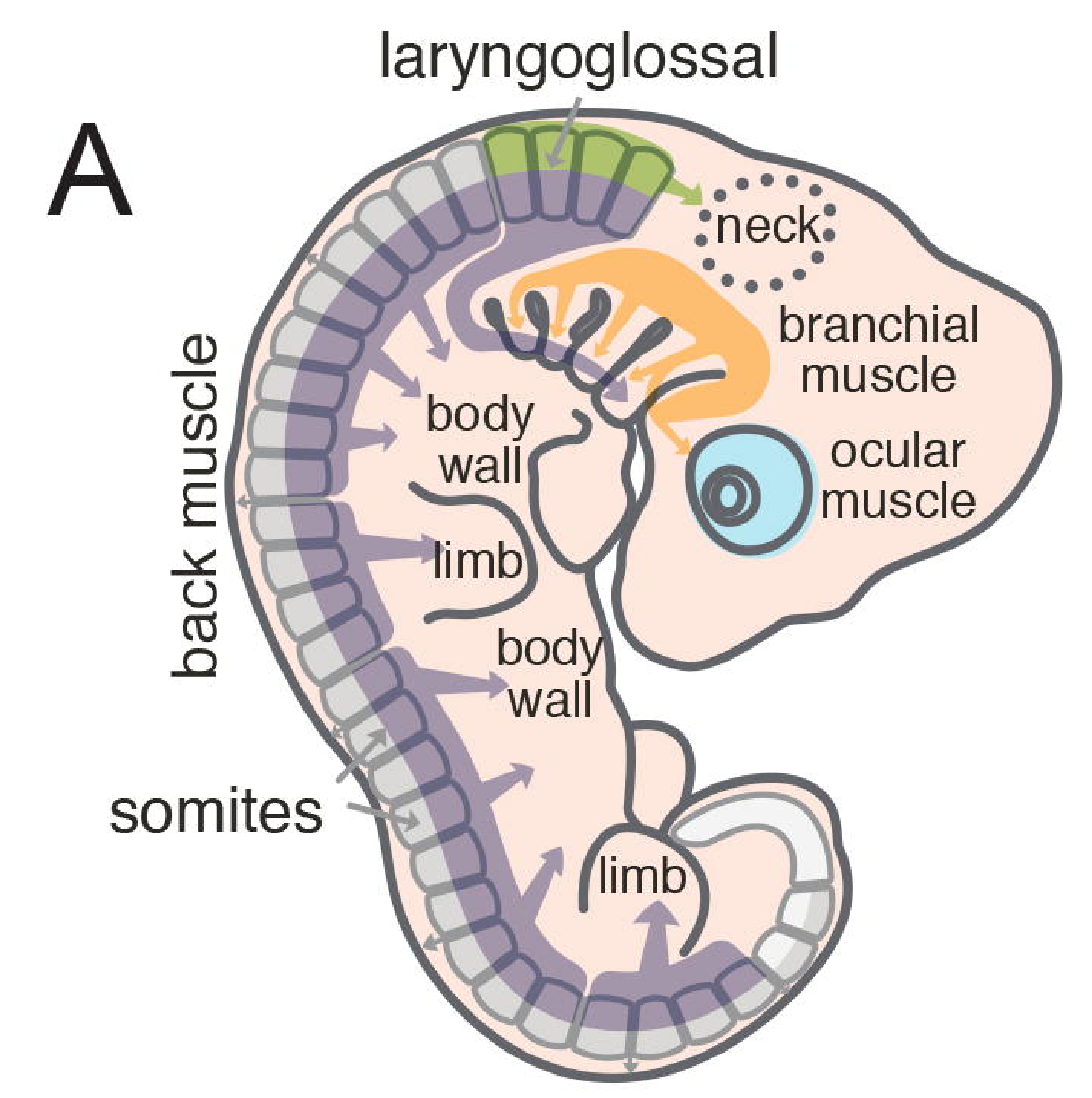

B

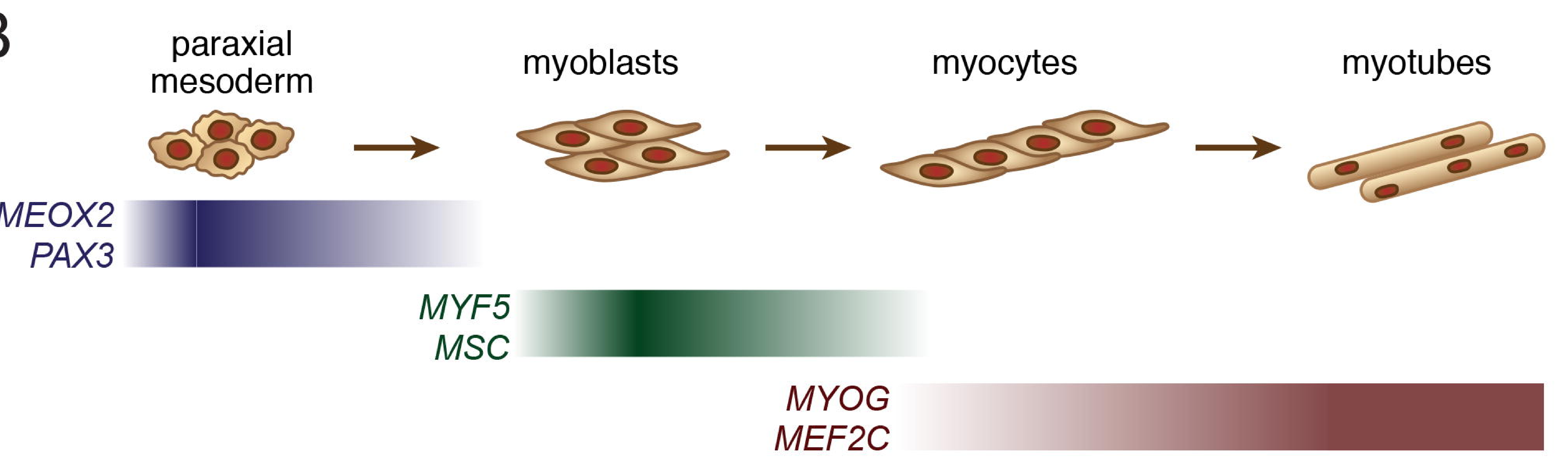

\section{SJRHB030680_R1(ERMS)}
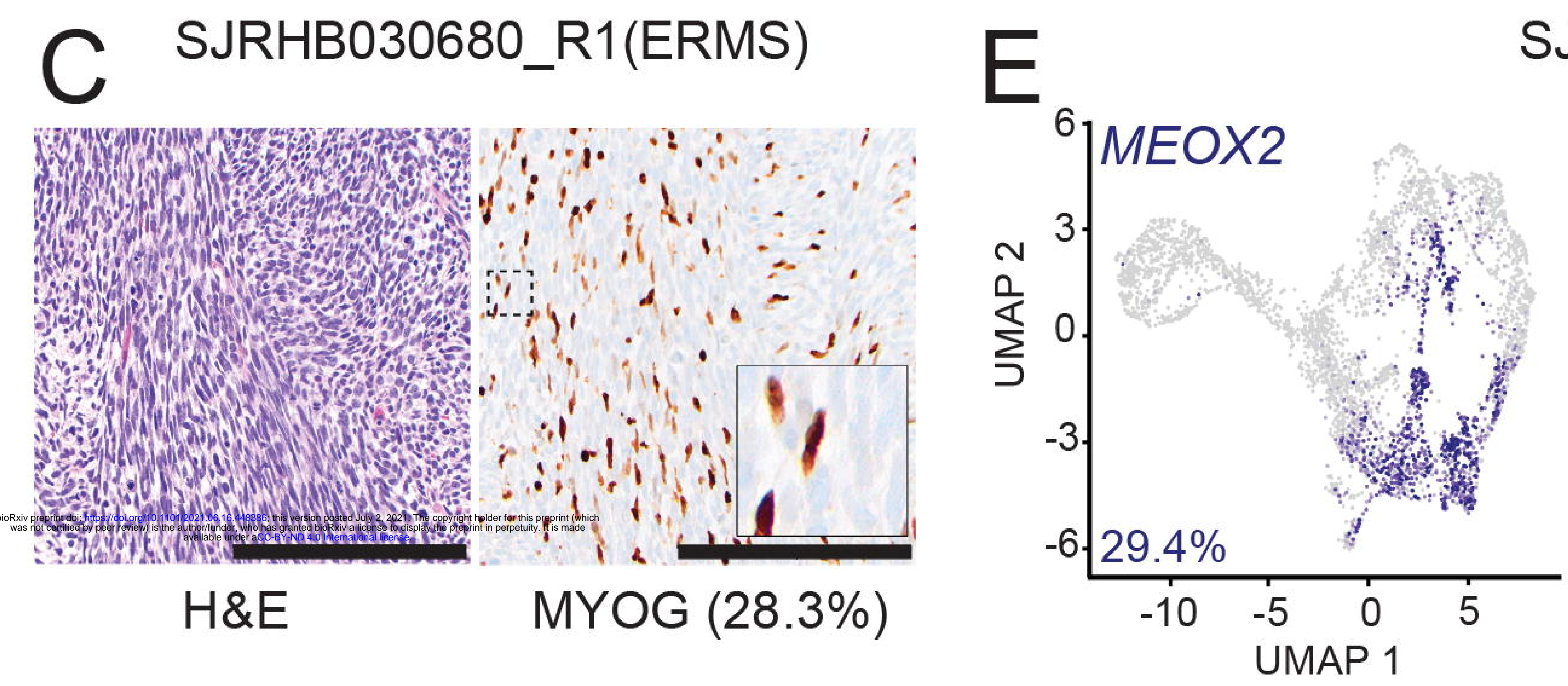

\section{SJRHB030680_R1(ERMS)}

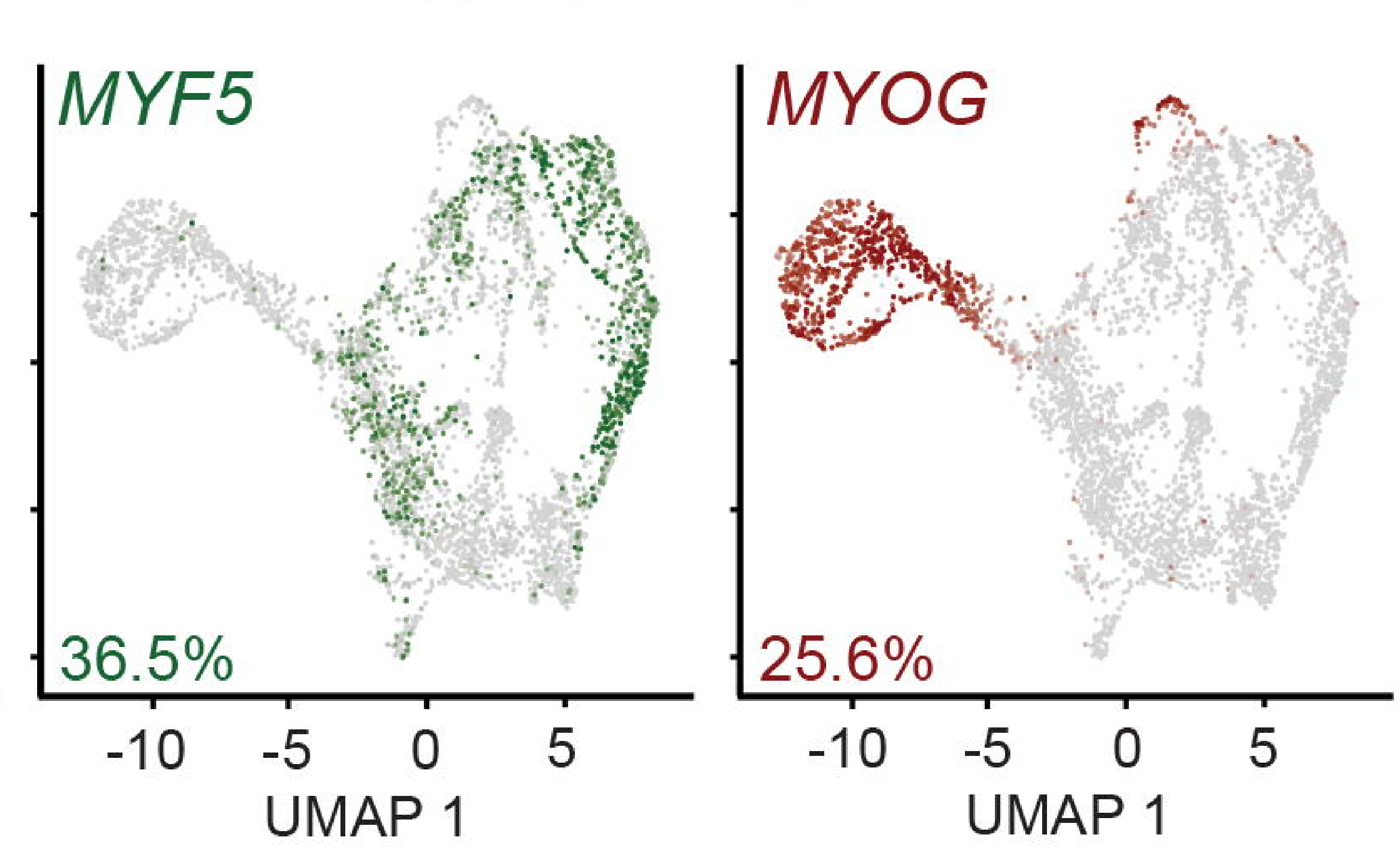
D SJRHB031320_D1 (ARMS)

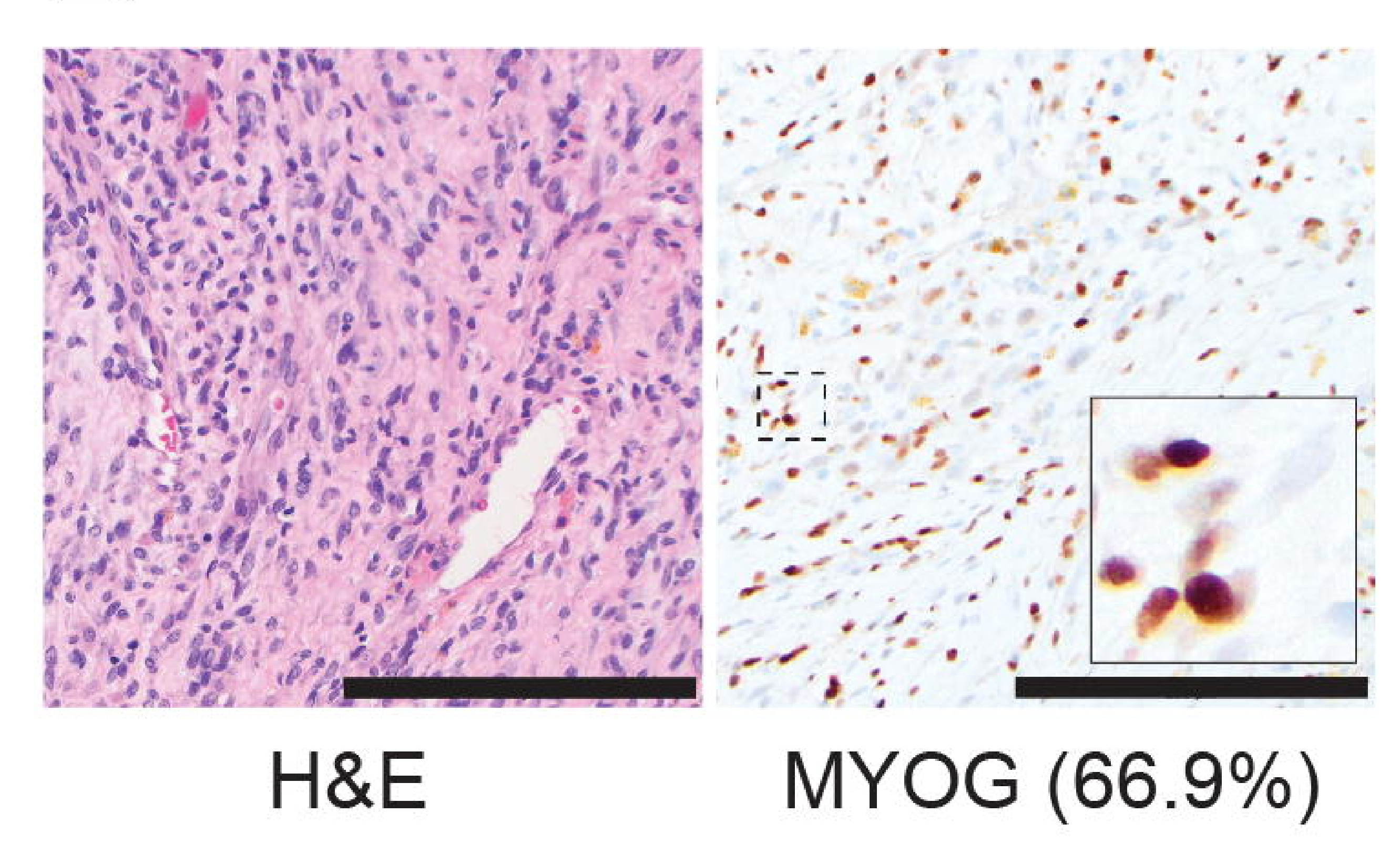

$\mathrm{F}_{50}$

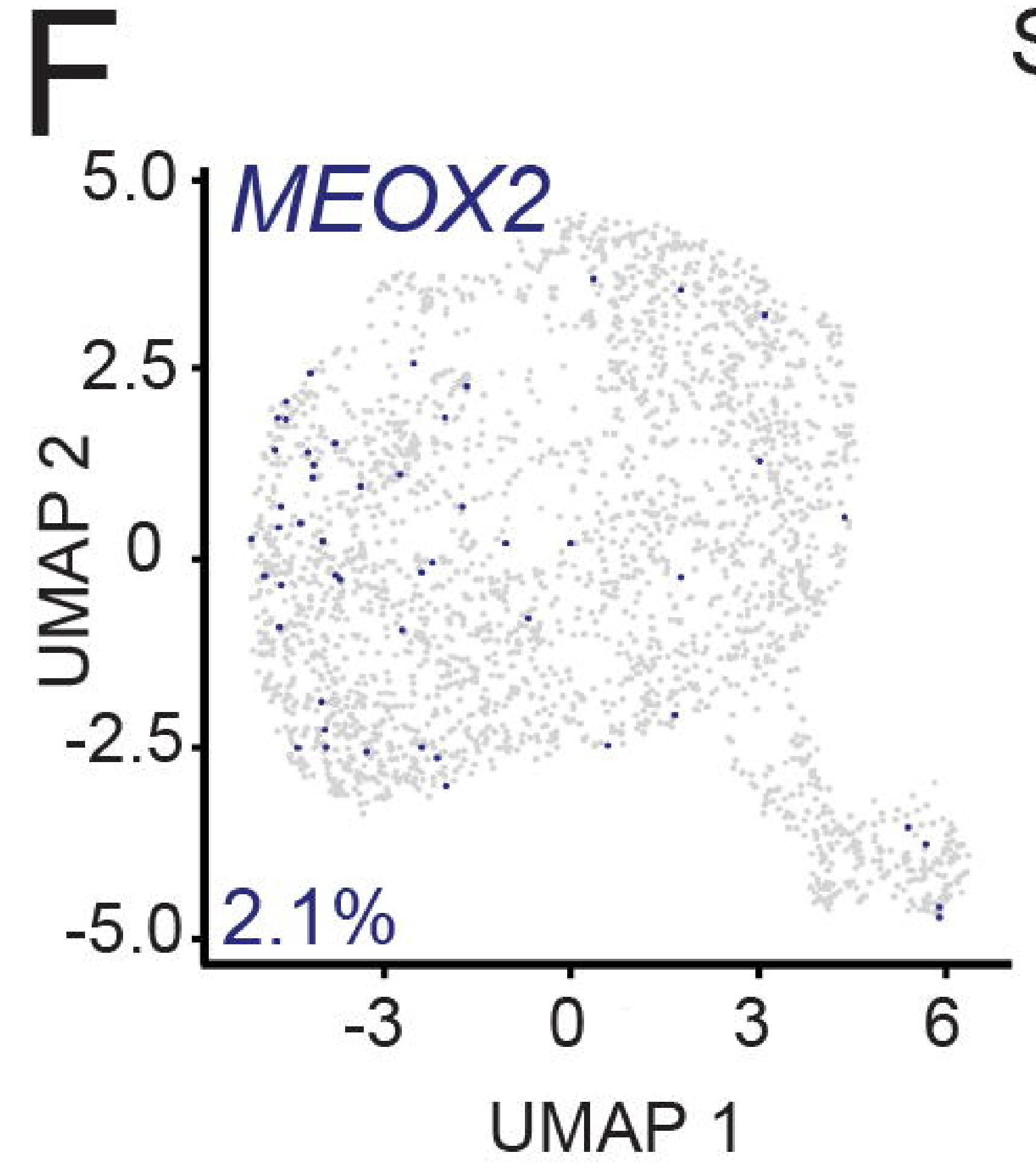

SJRHB031320_D1 (ARMS)
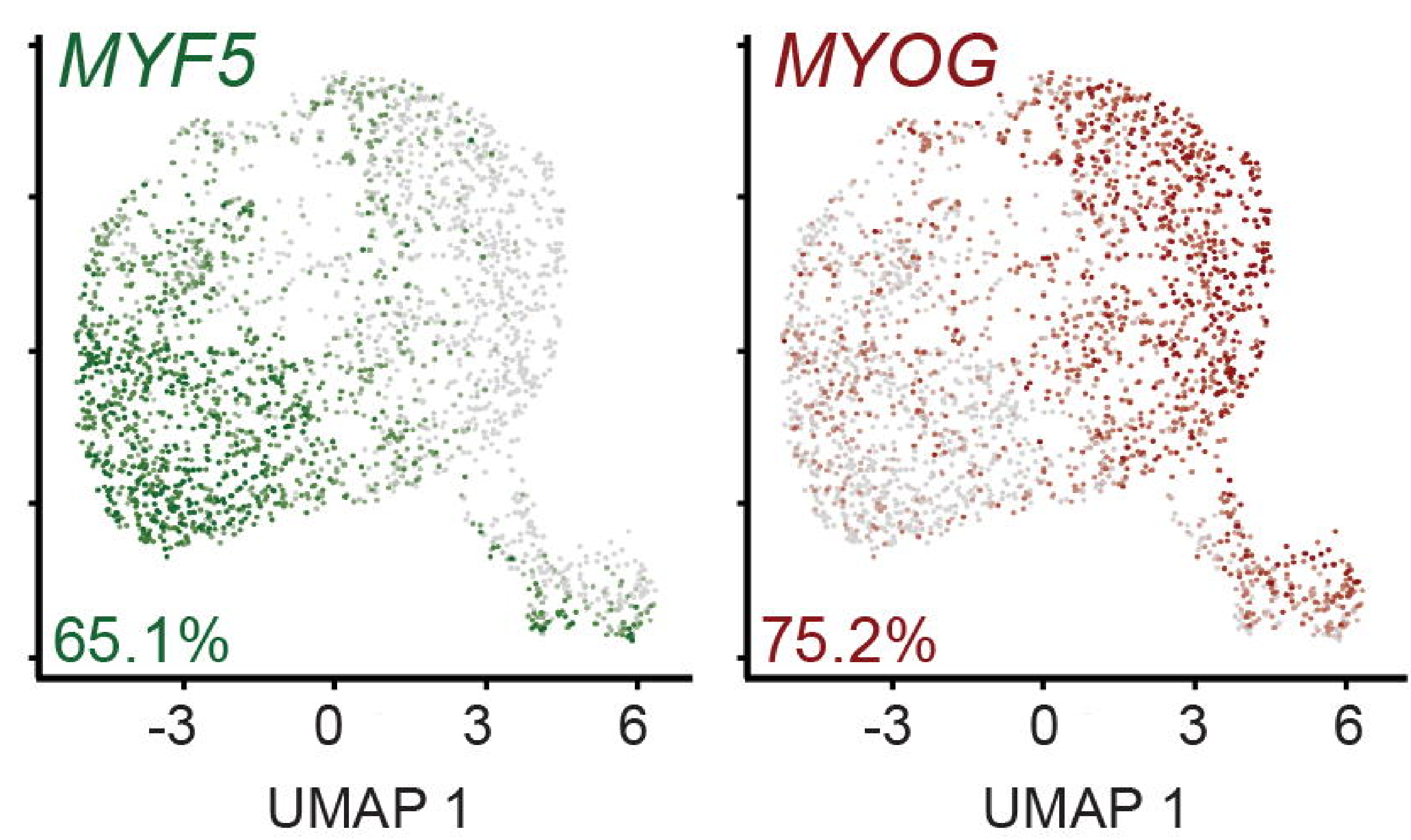

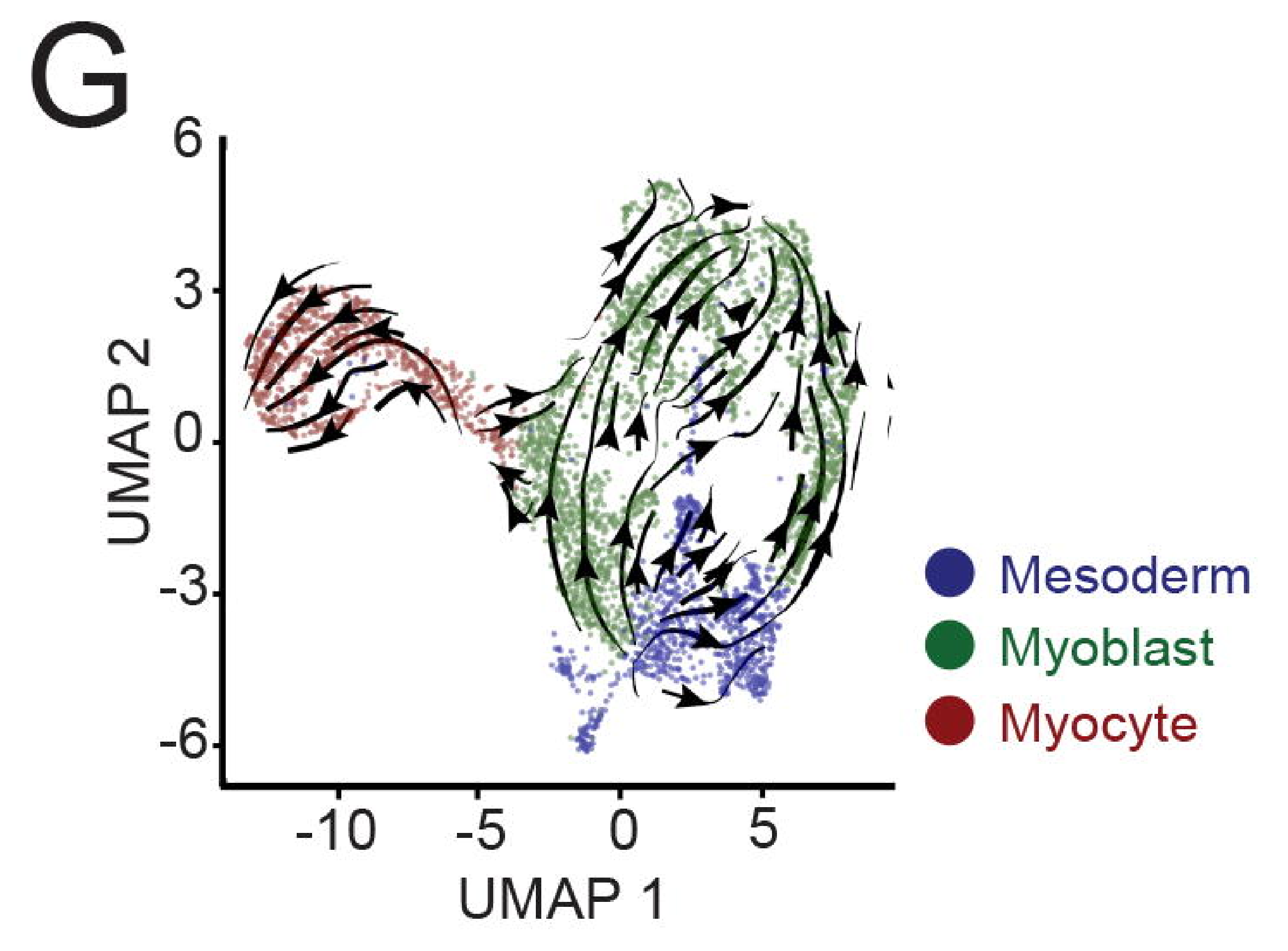

$\mathrm{H}$

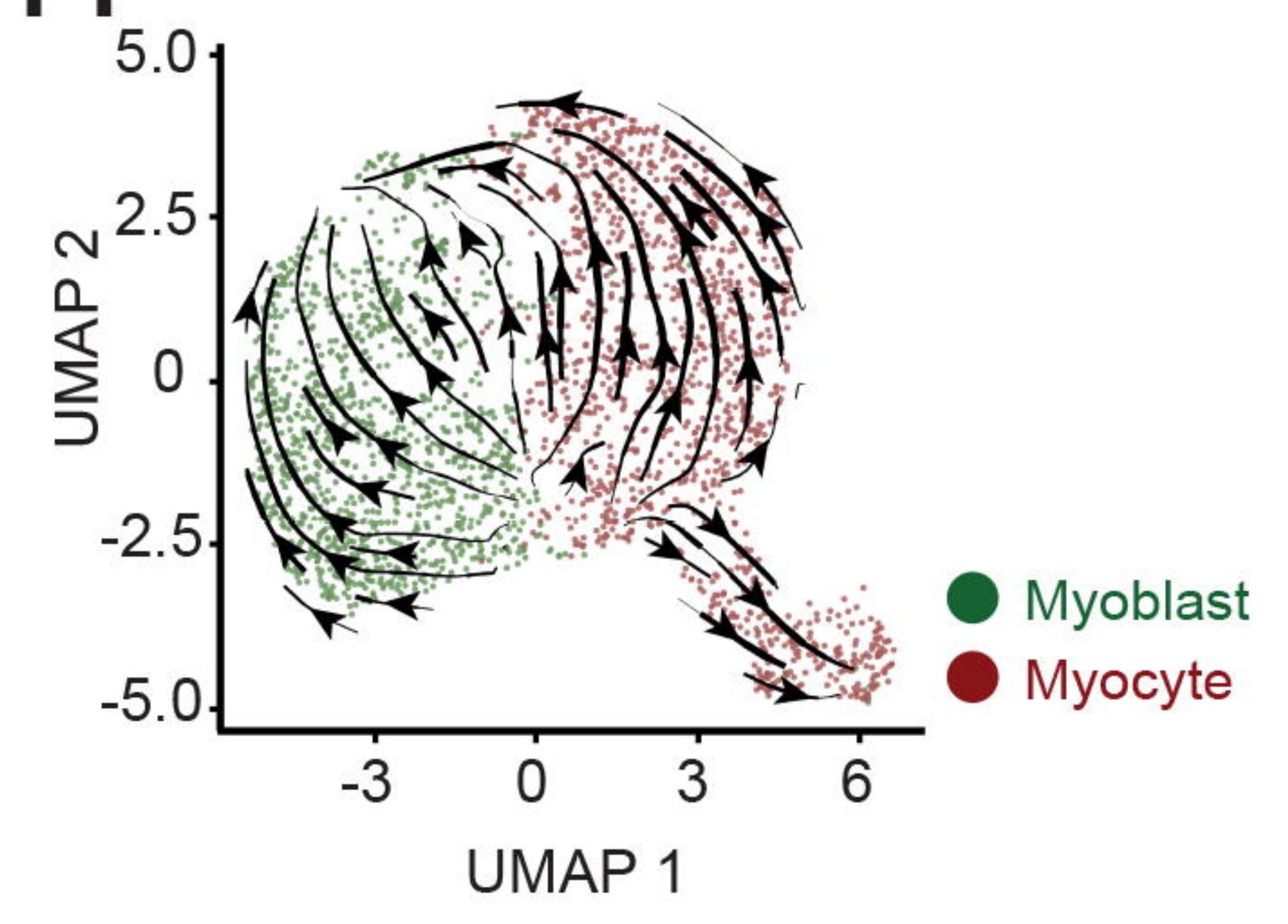


Figure 2
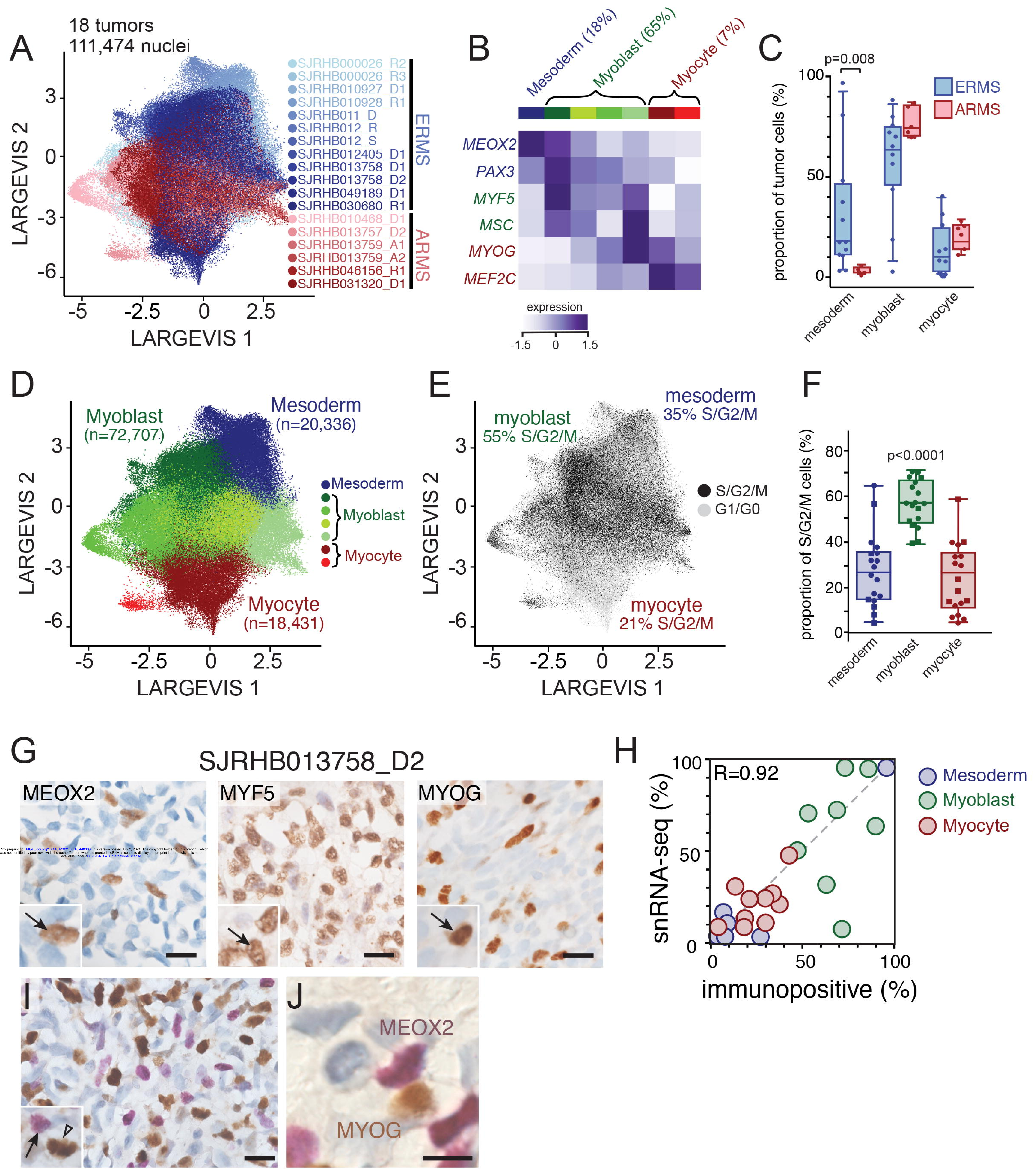
Figure 3

A murine embryogenesis

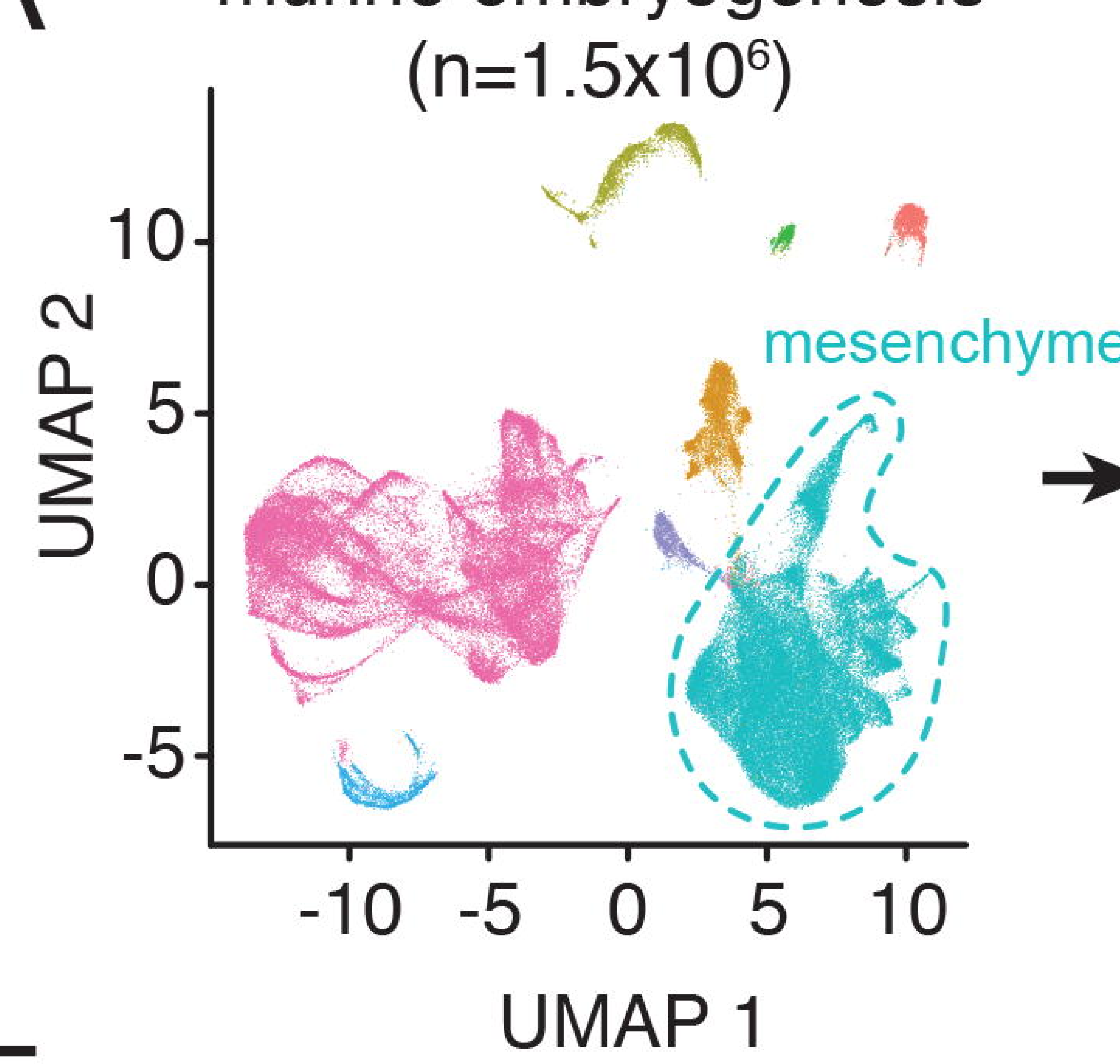

E

mouse myogenesis

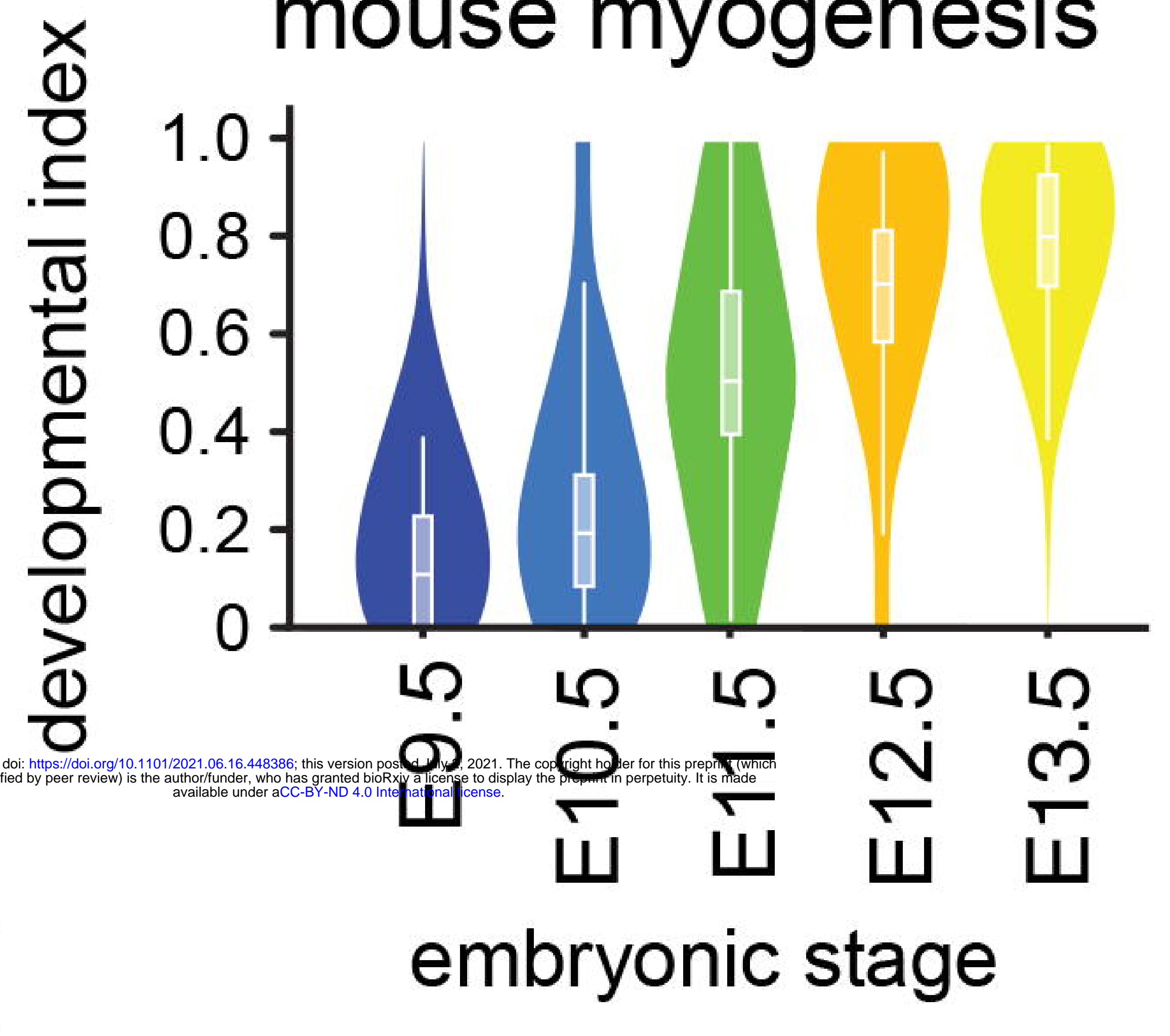

$\frac{x}{0}$

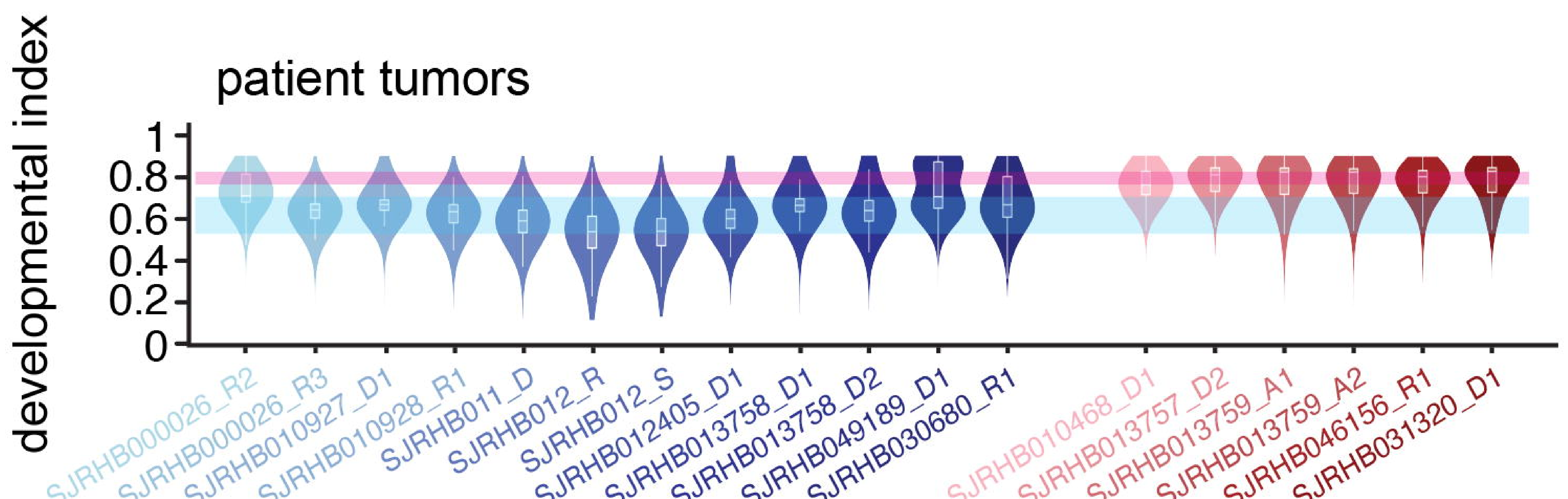

ERMS tumors
B mesenchymal trajectory

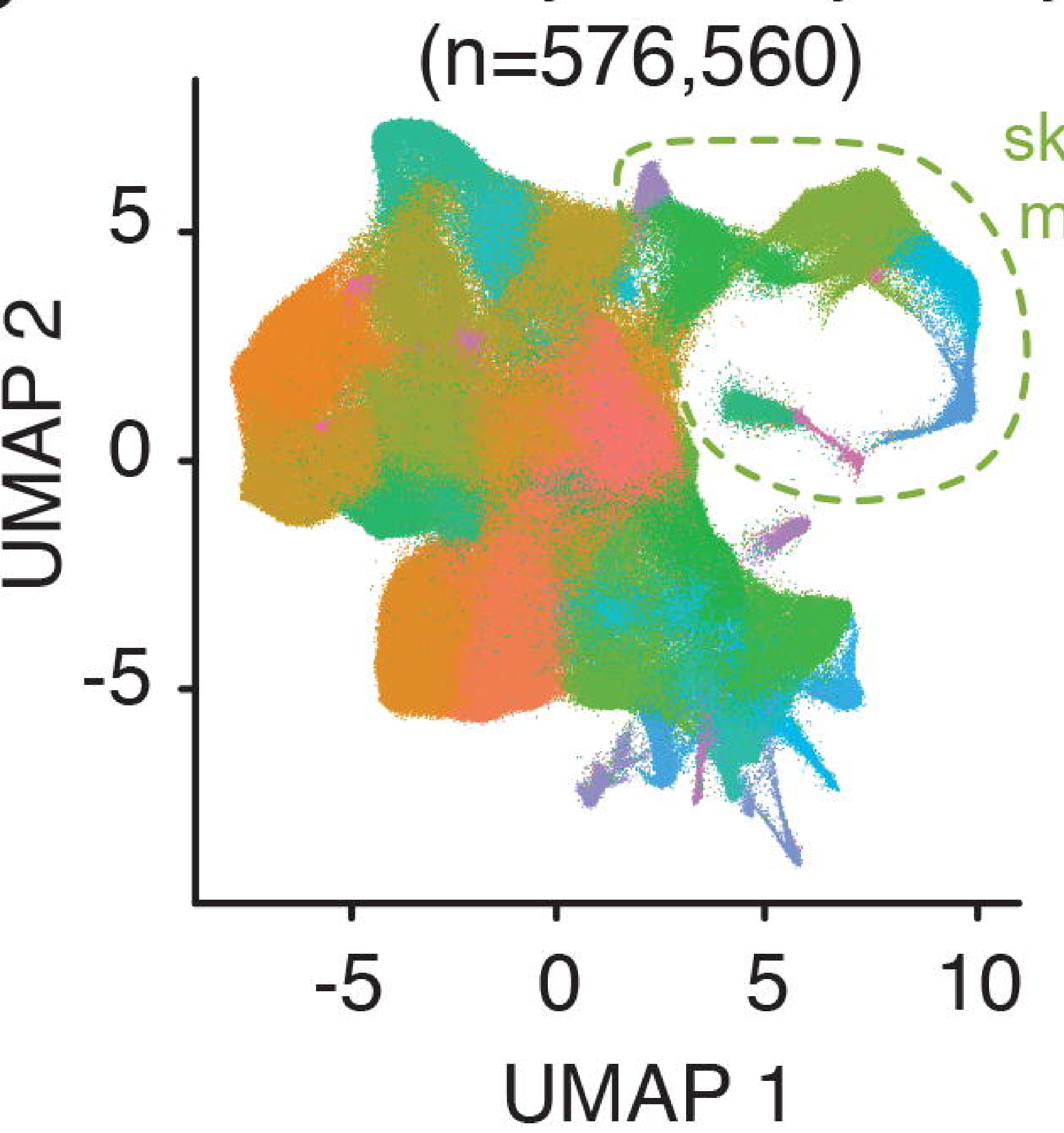

$\mathrm{F}$

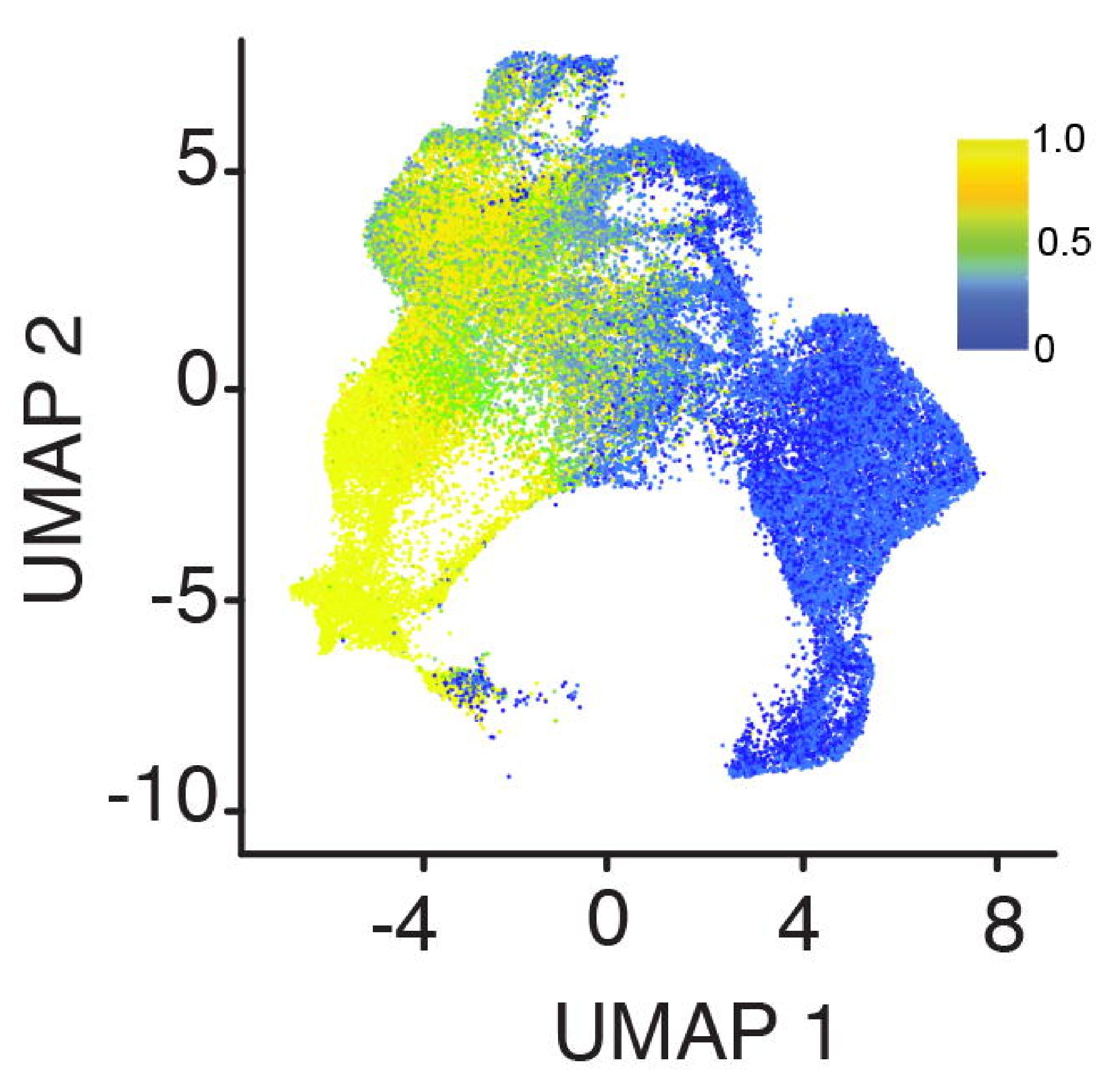

C skeletal muscle sub-trajectory

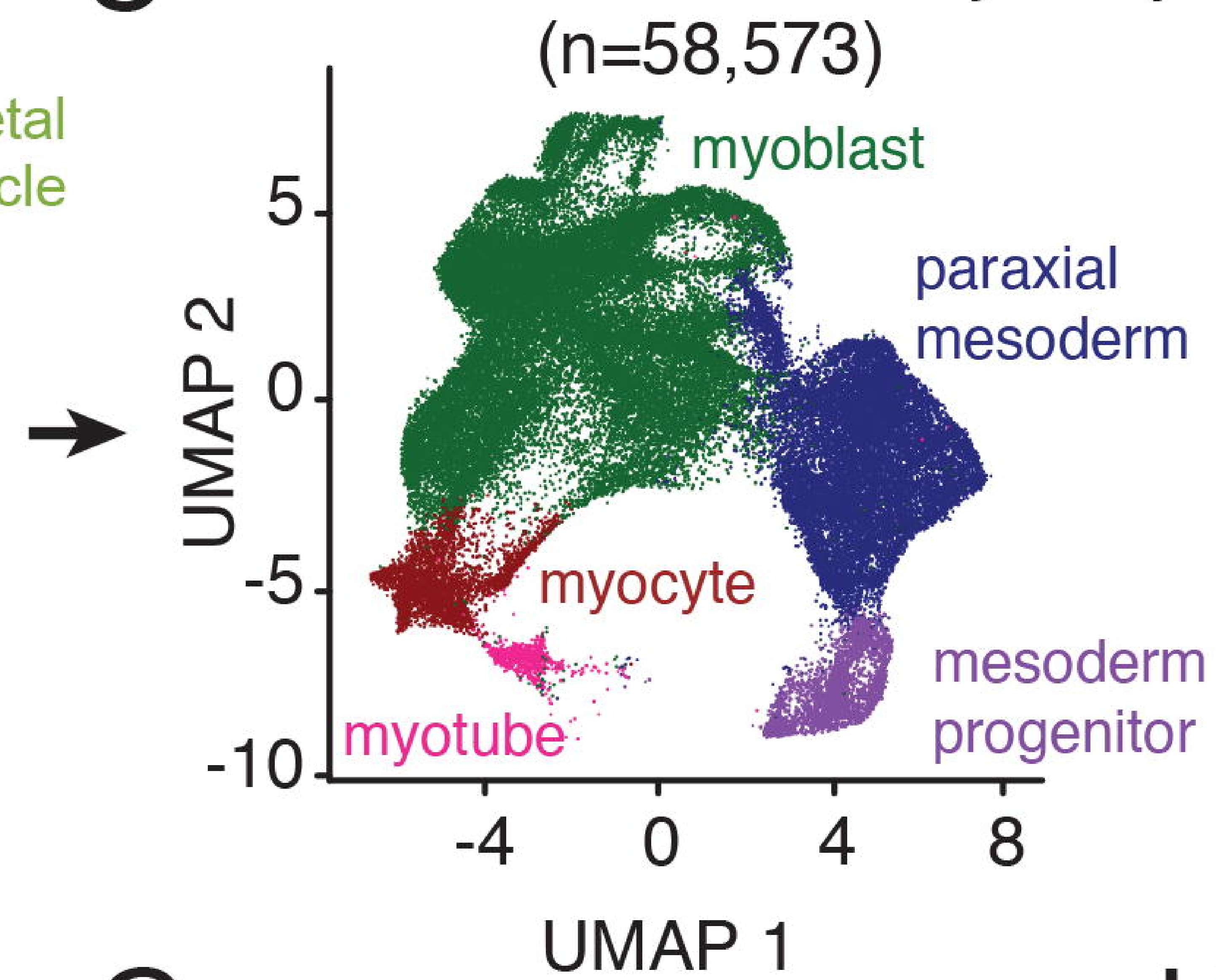

$G$

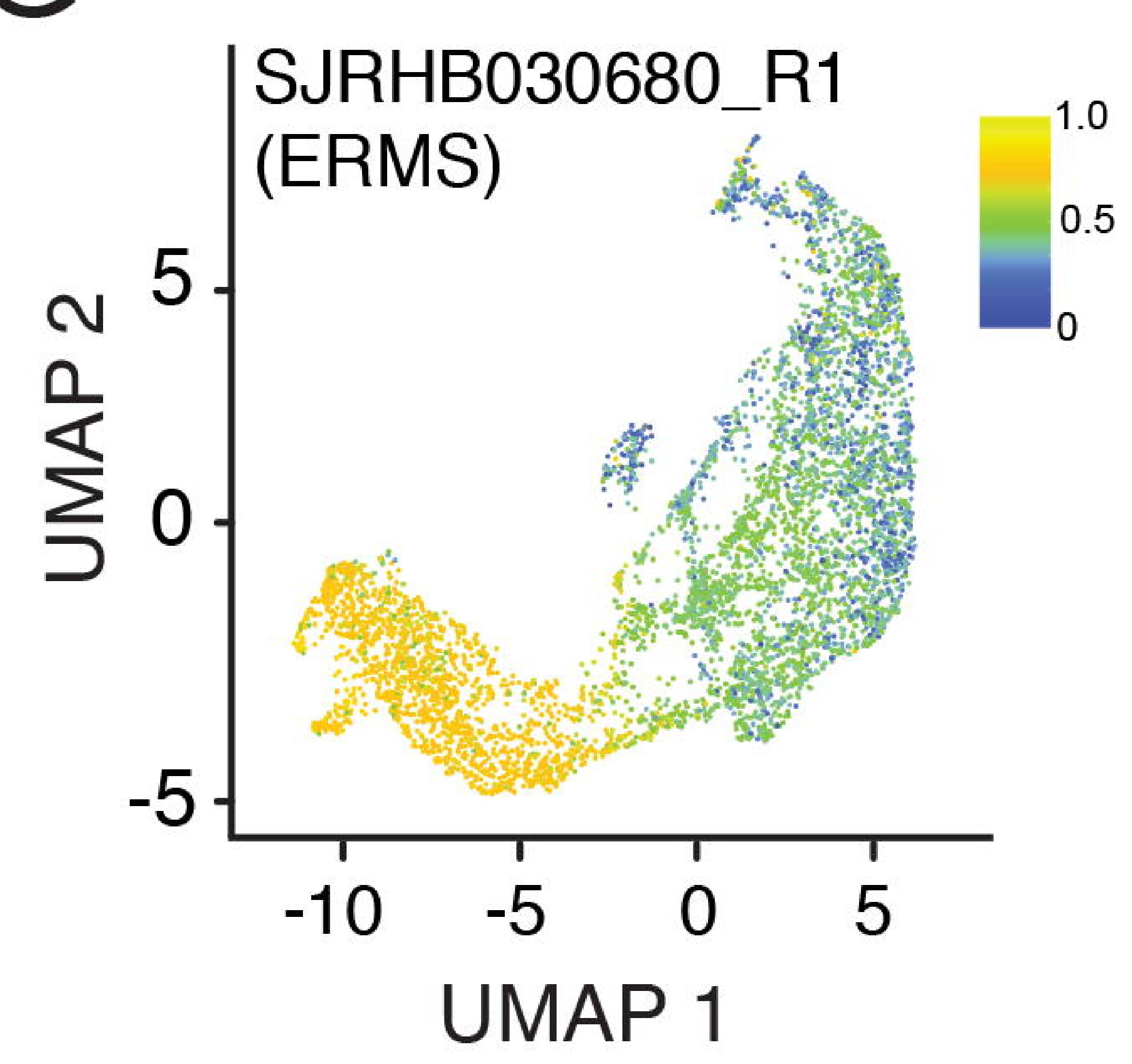

$\mathrm{H}$
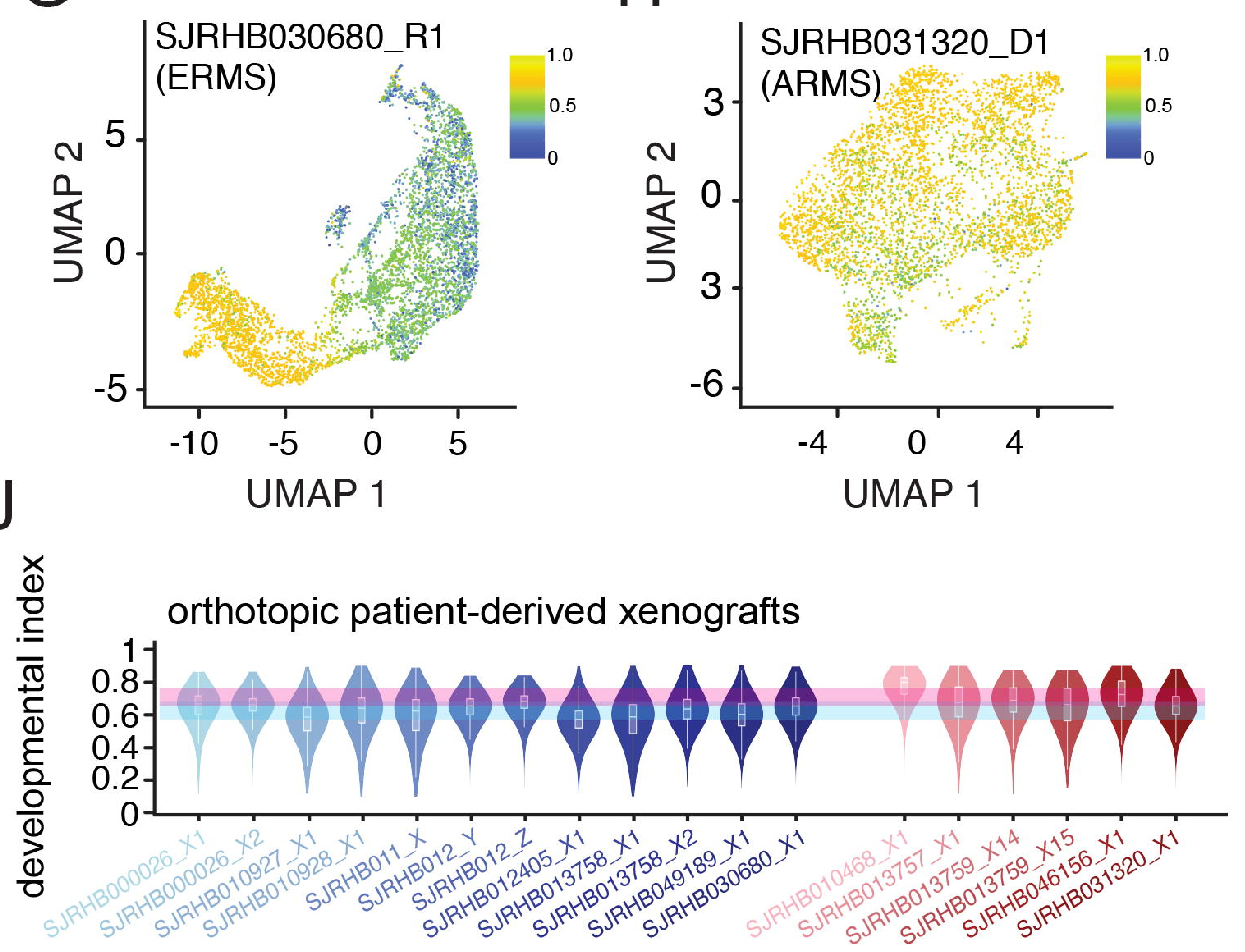

ERMS O-PDXs

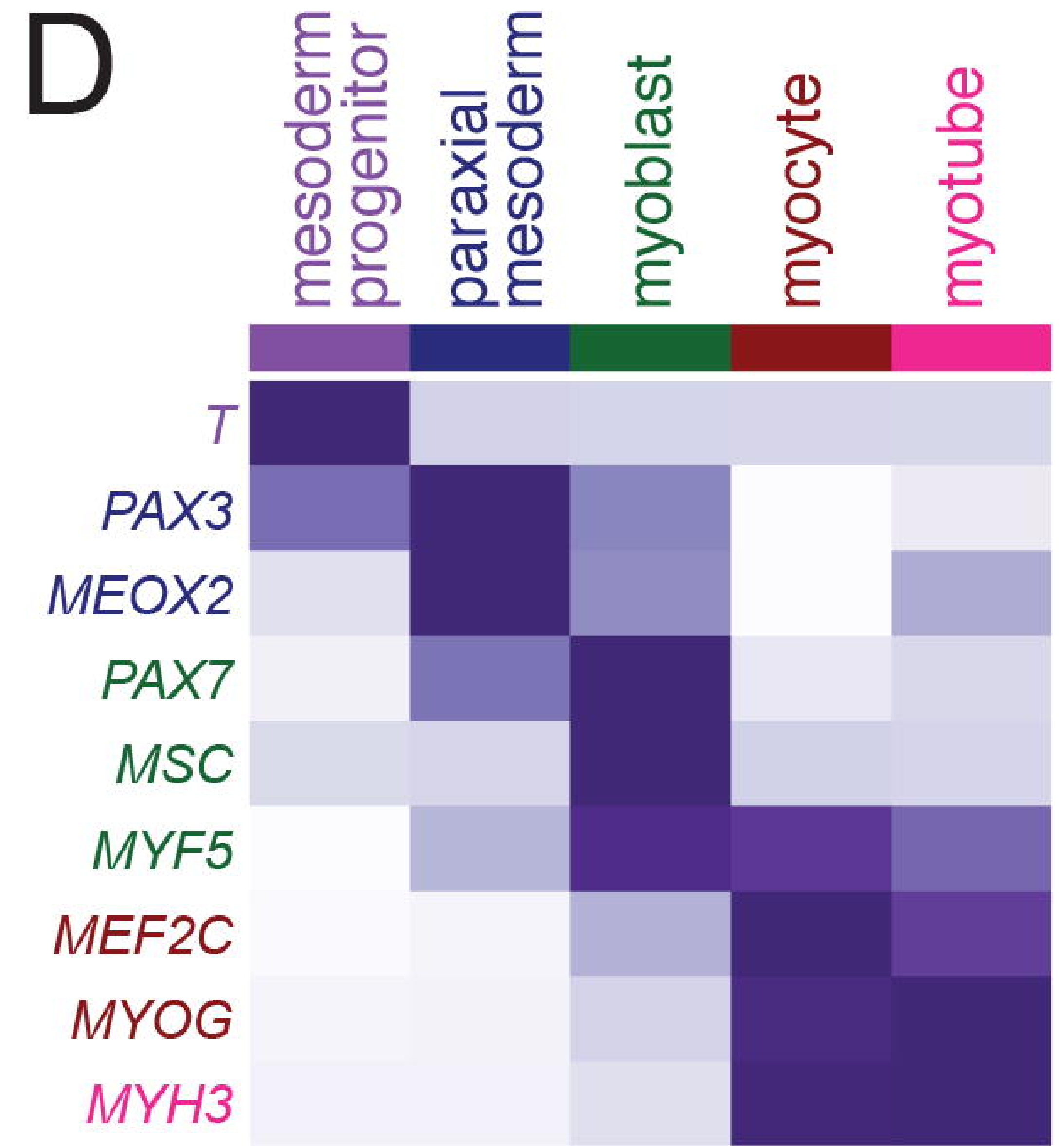

ARMS O-PDXs 
Figure 4

mesoderm myoblasts myocytes
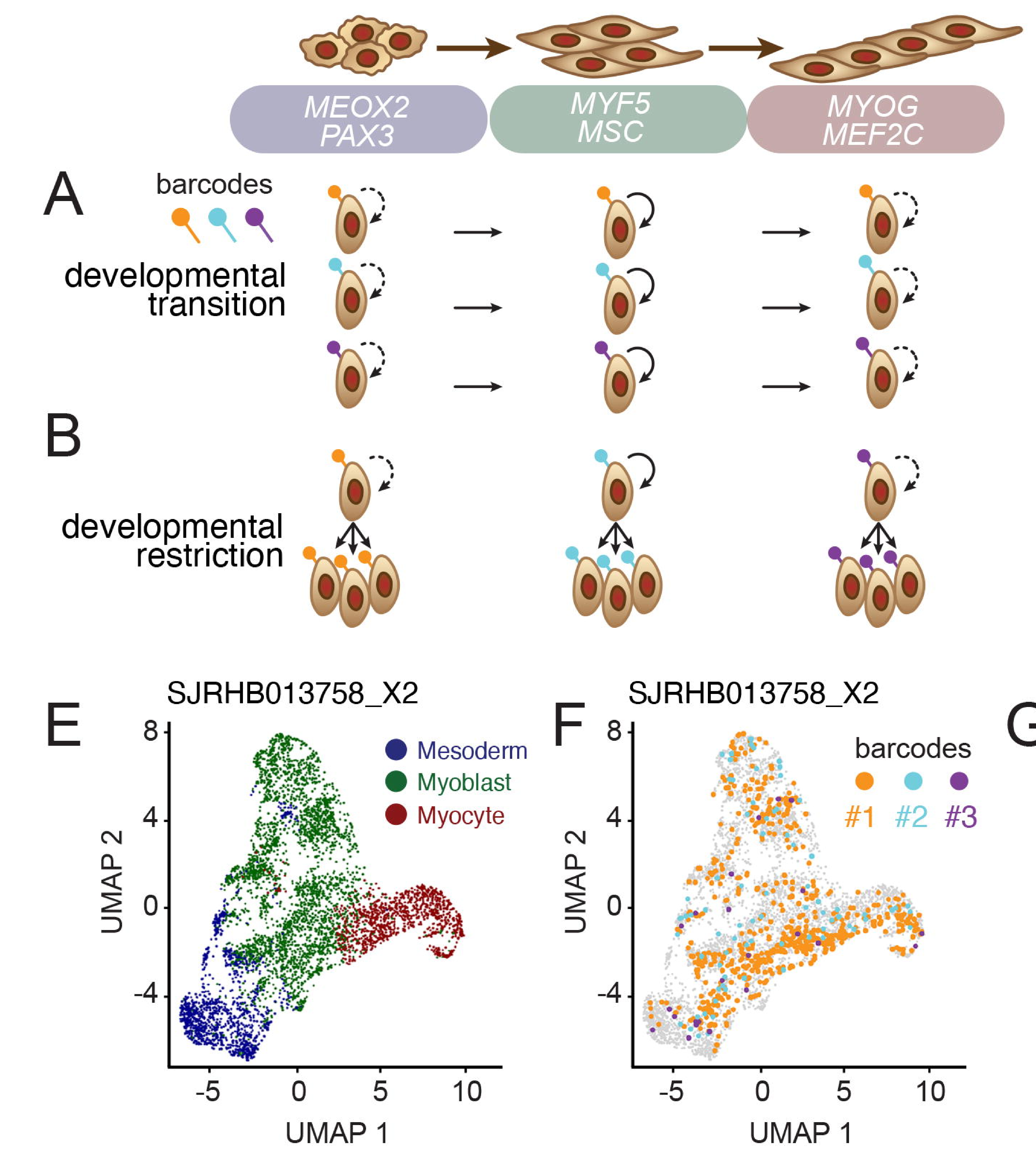

A barcodes

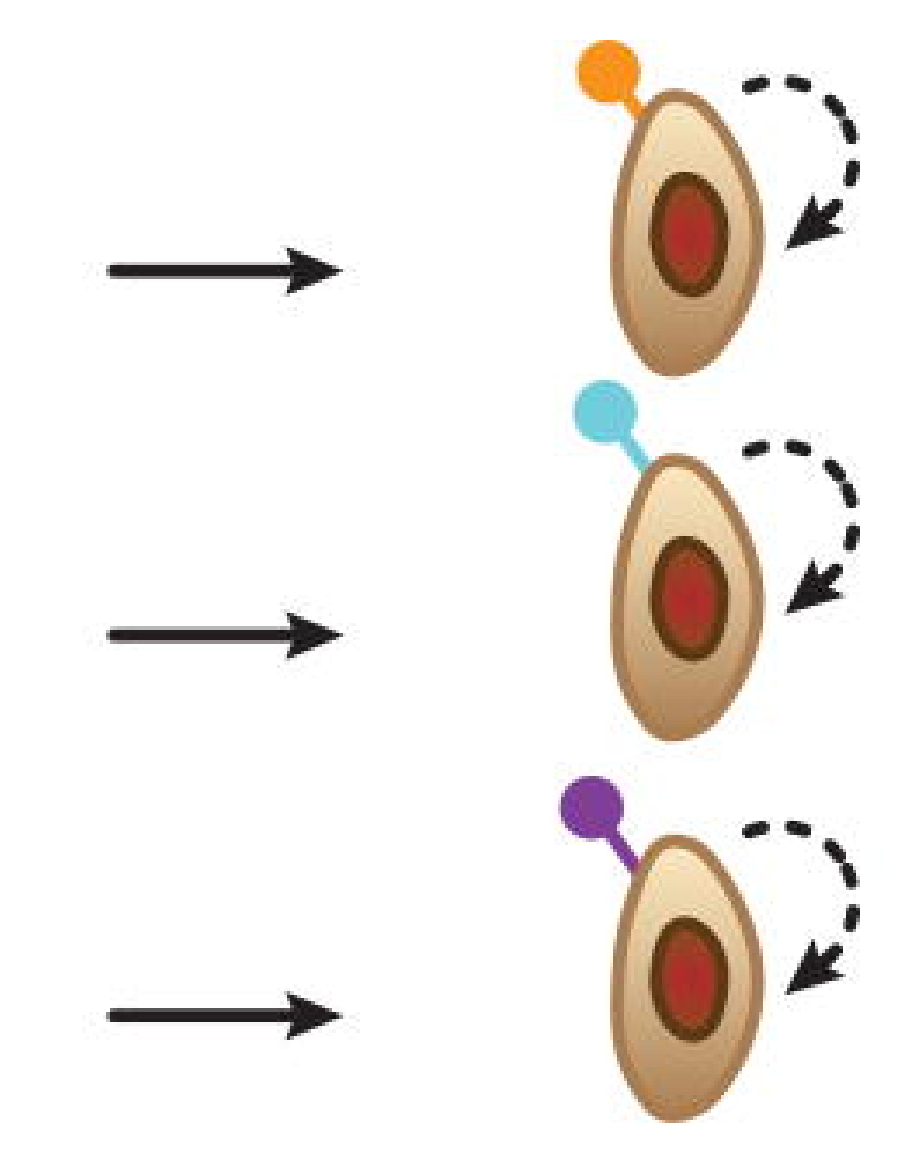

C
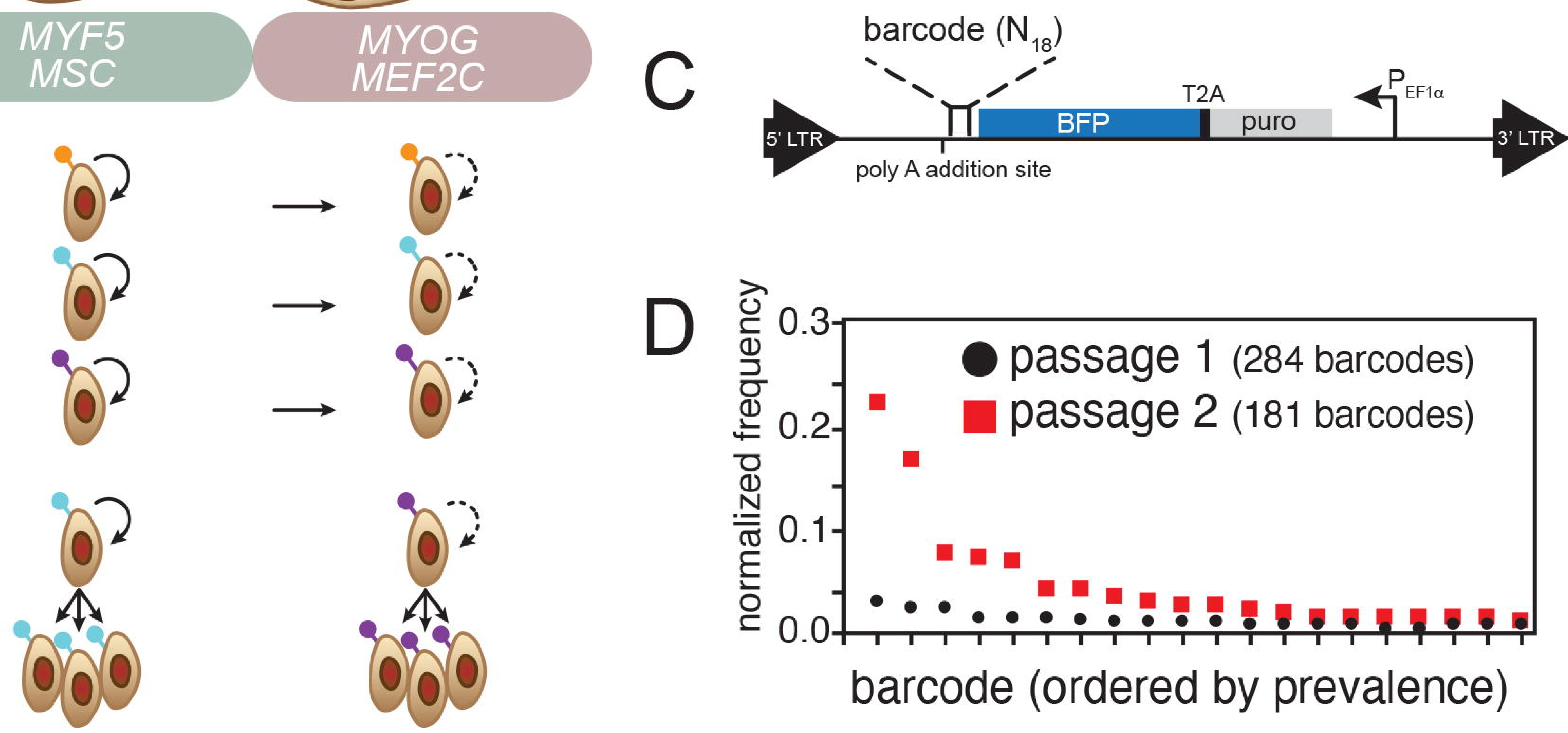

$\mathrm{D}$
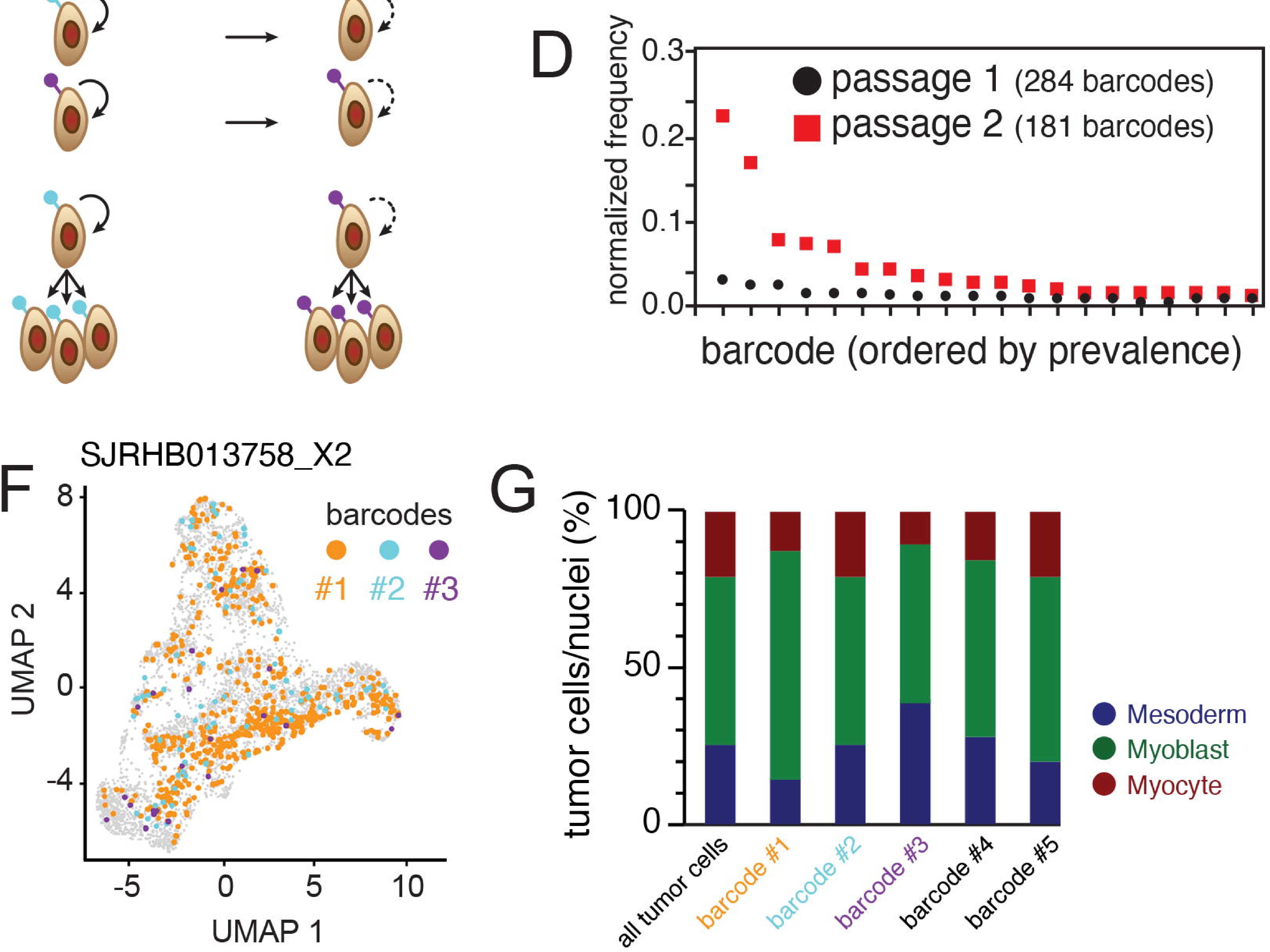

$\mathrm{G}$

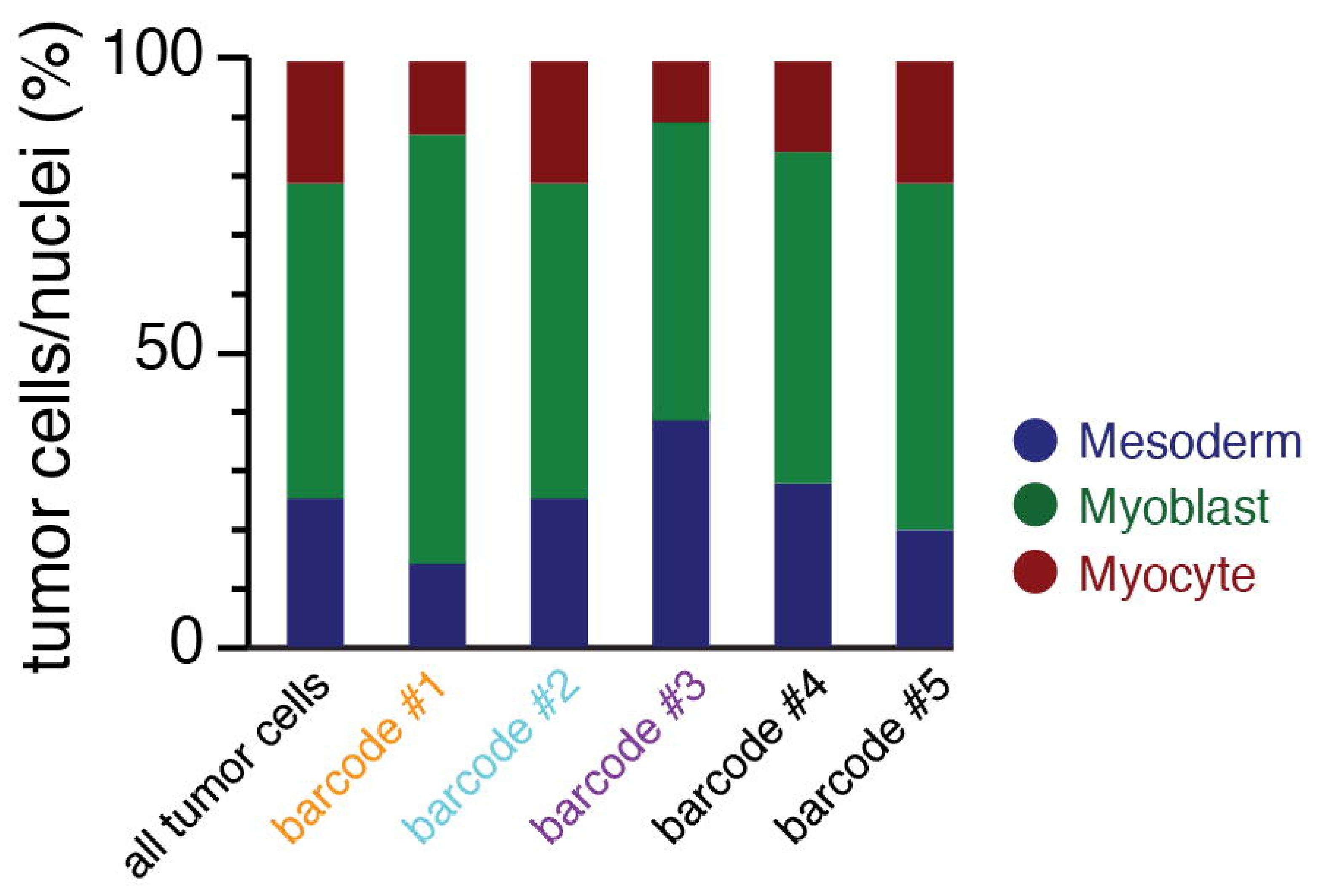

\section{MYOD1}

$\mathrm{H}$
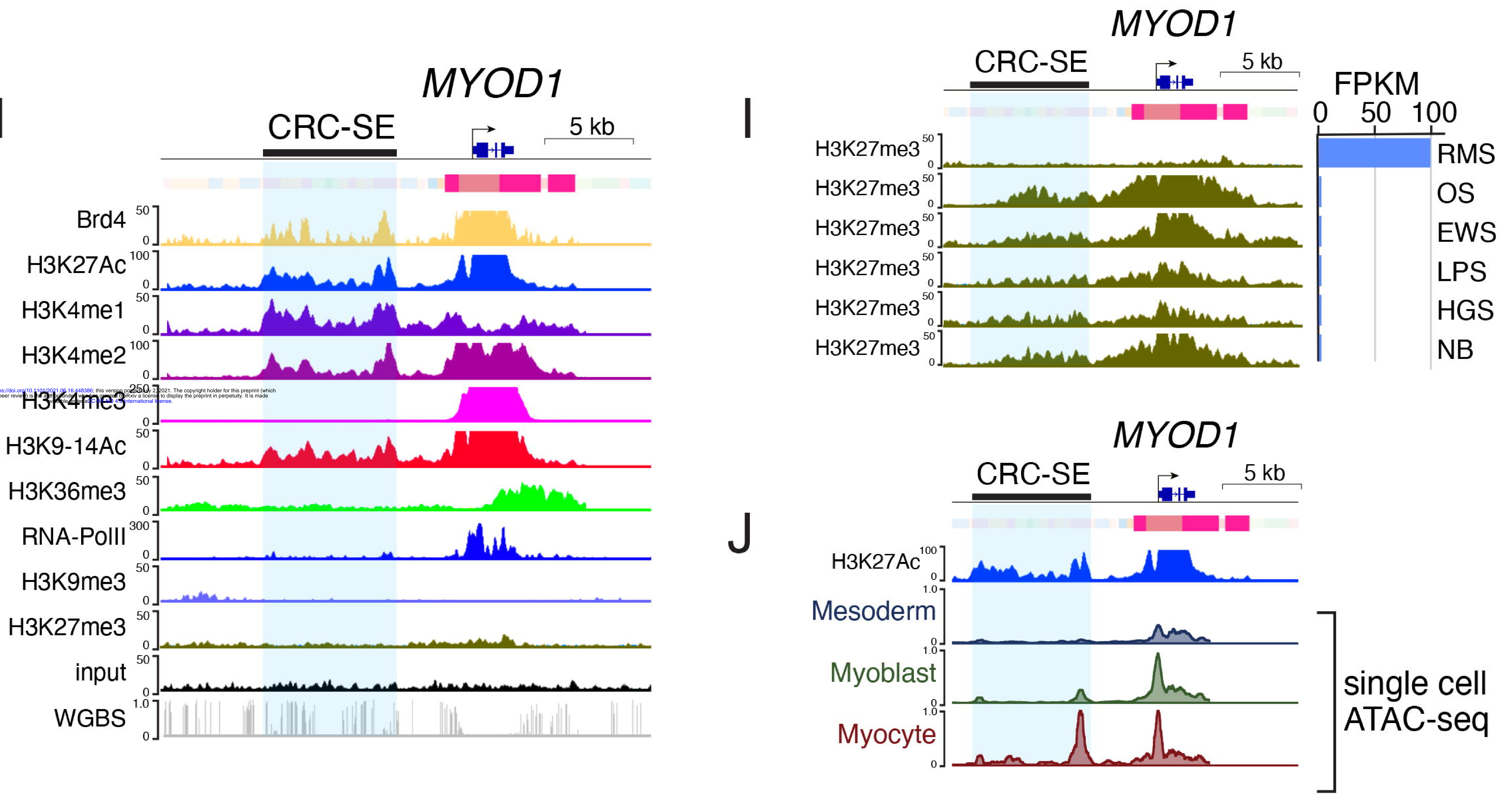
Figure 5

A

A patient tumor

B

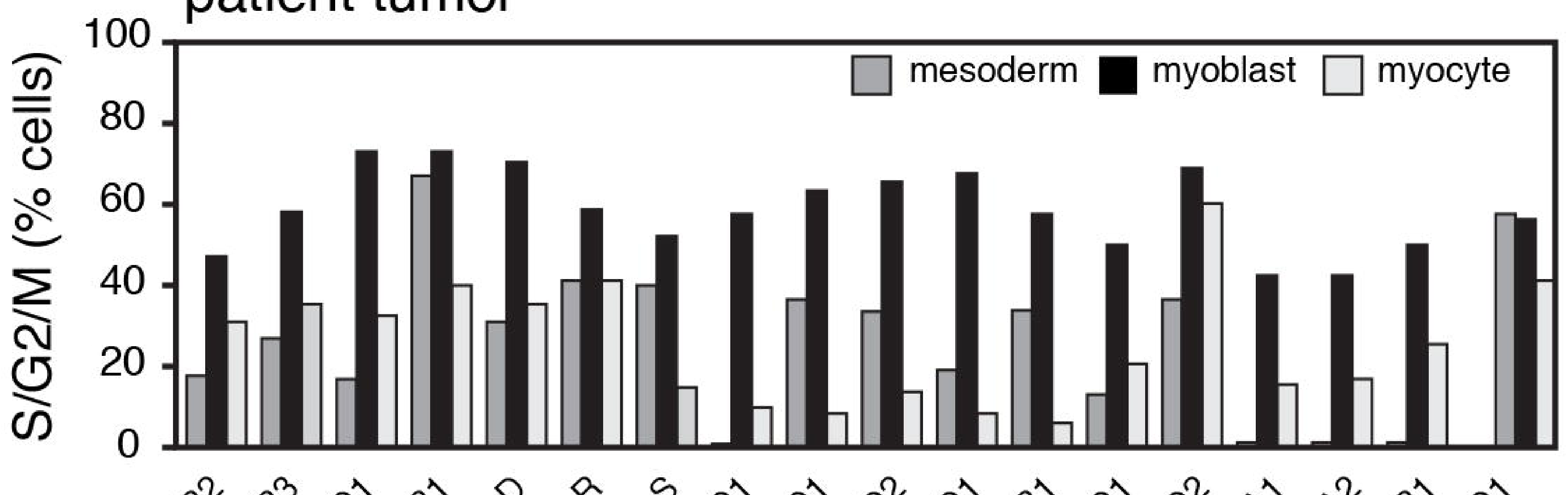

100 O-PDX

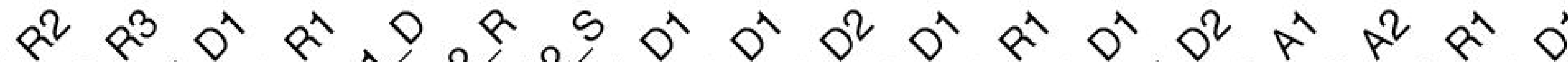

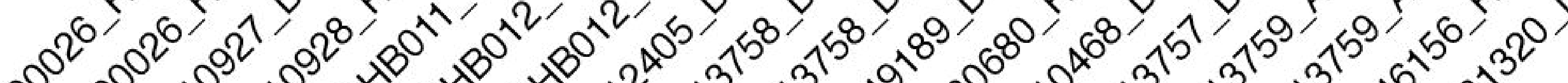

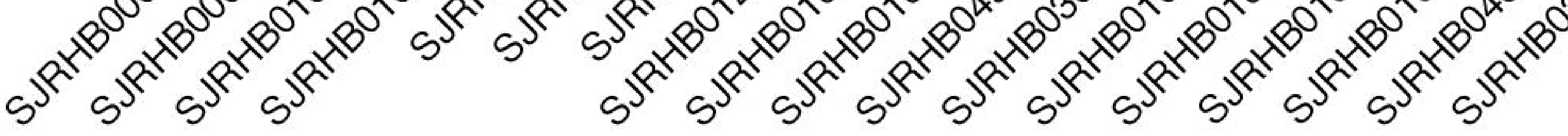
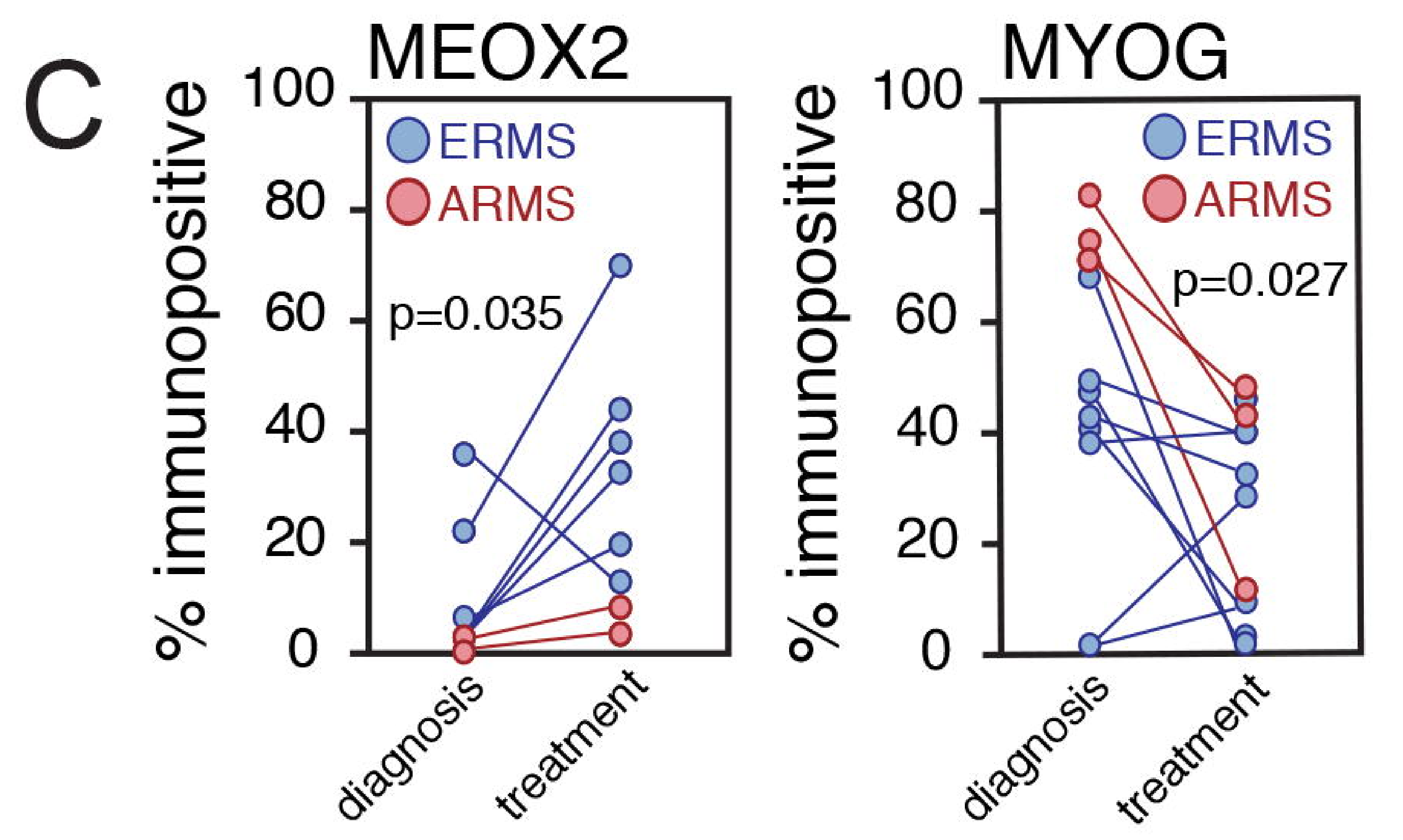

$\mathrm{D}$

\begin{tabular}{lll}
\hline VCR & IRN $\quad \nabla_{\text {biopsy }}$ \\
\hline
\end{tabular}

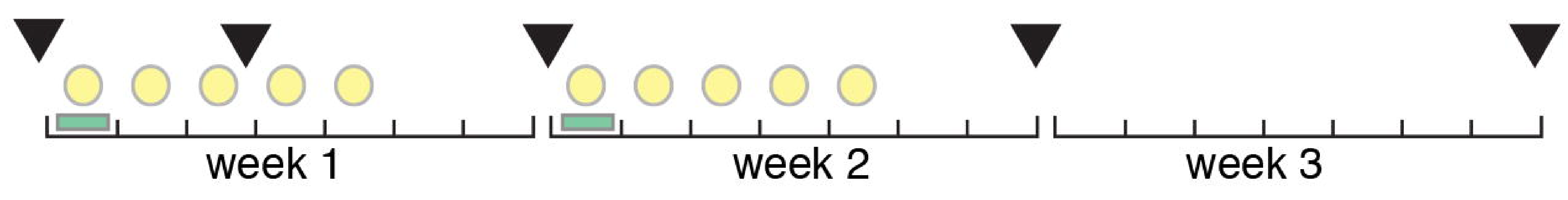

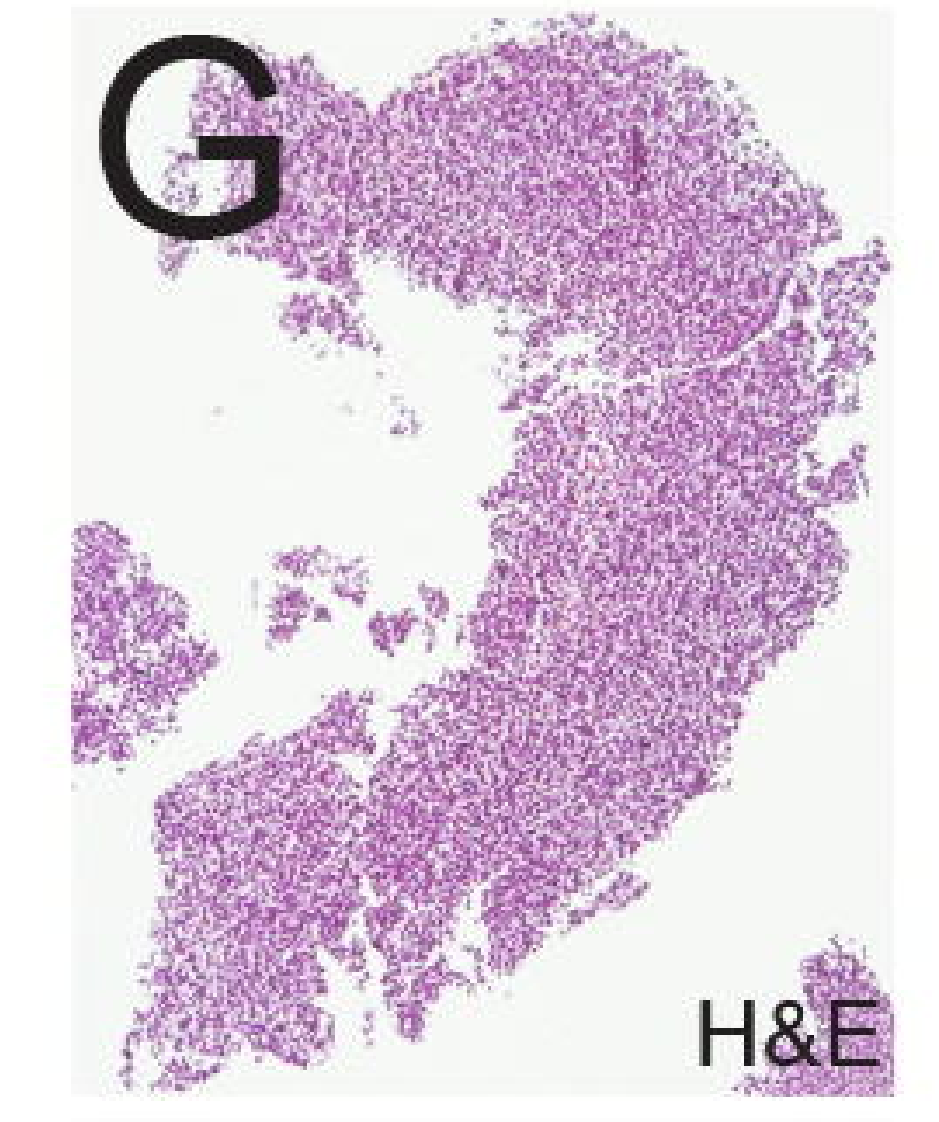

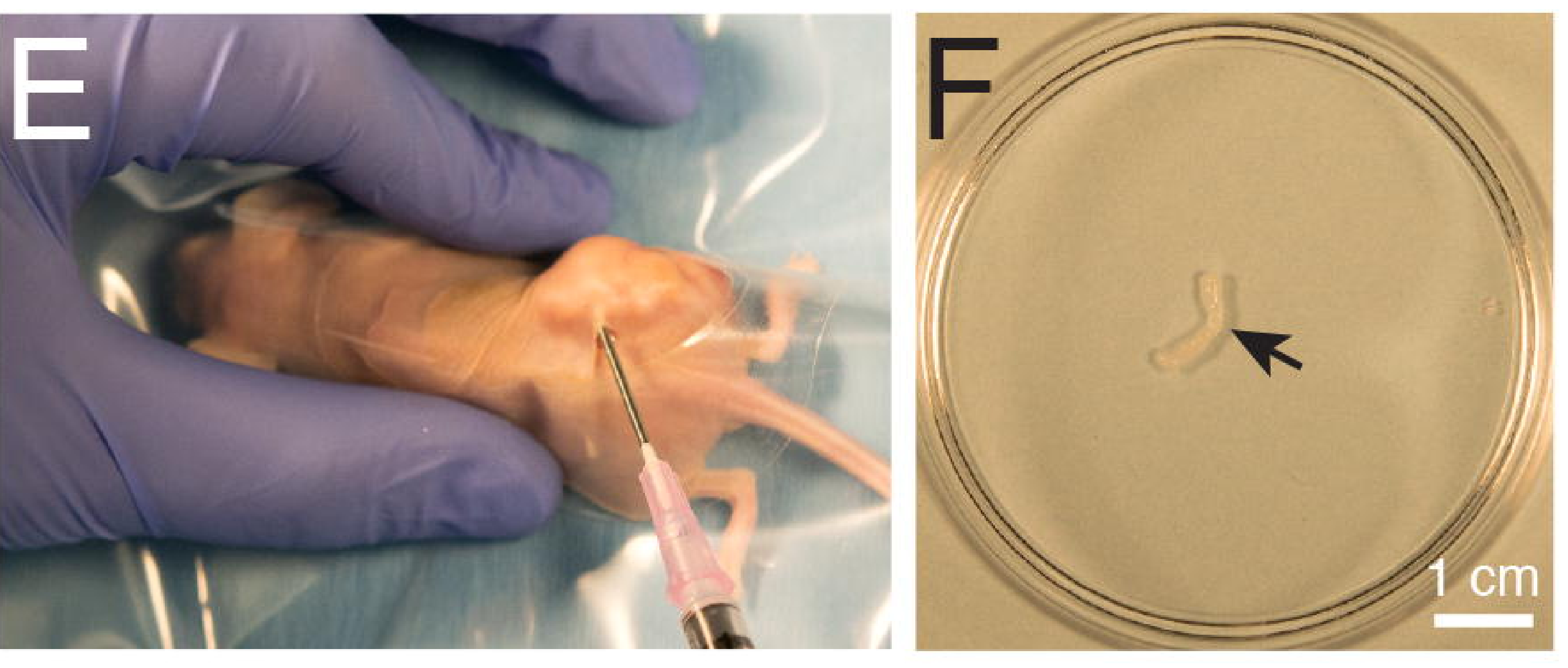

H
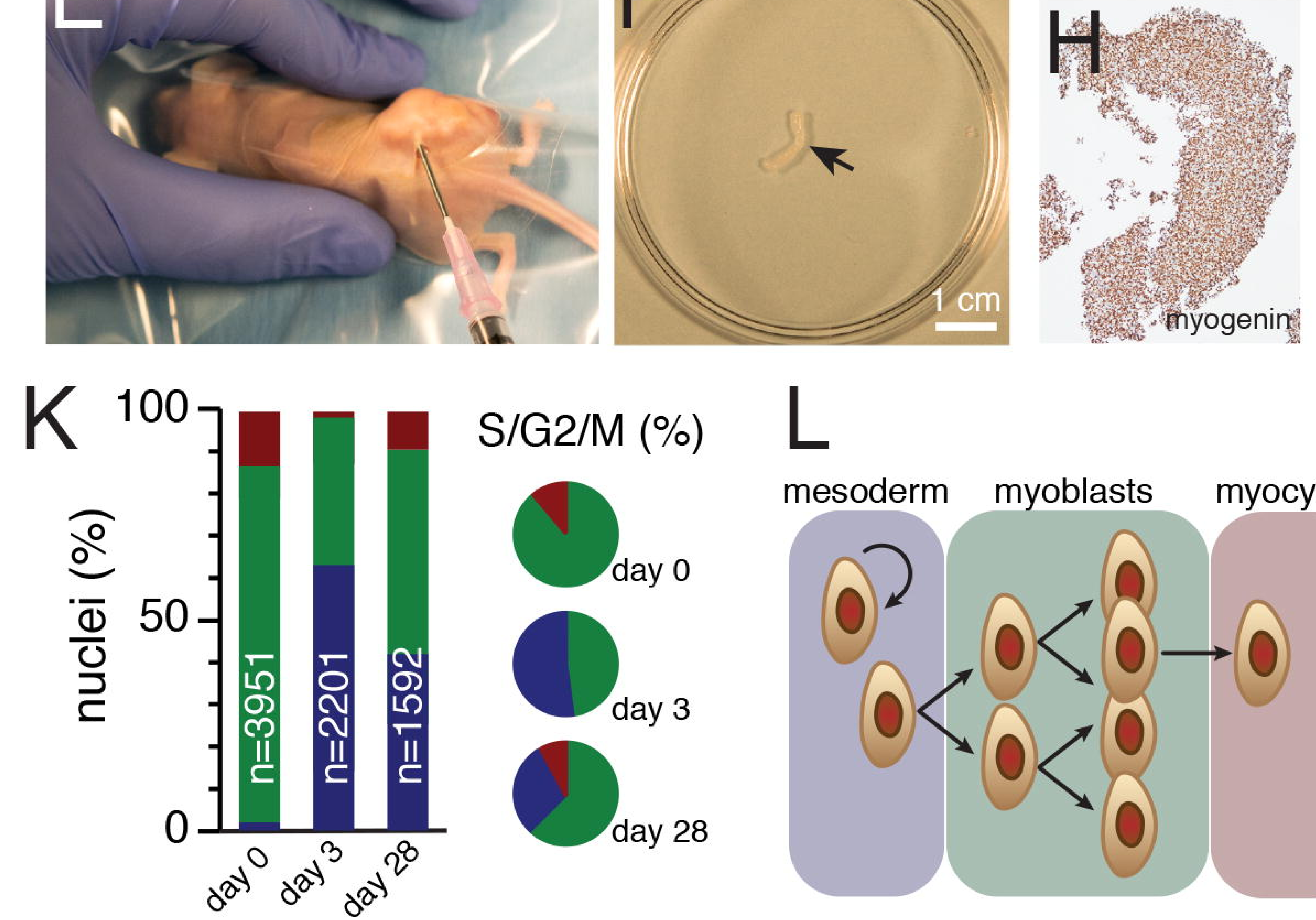

Mesoderm Myoblast Myocyte

$\mathrm{O}$
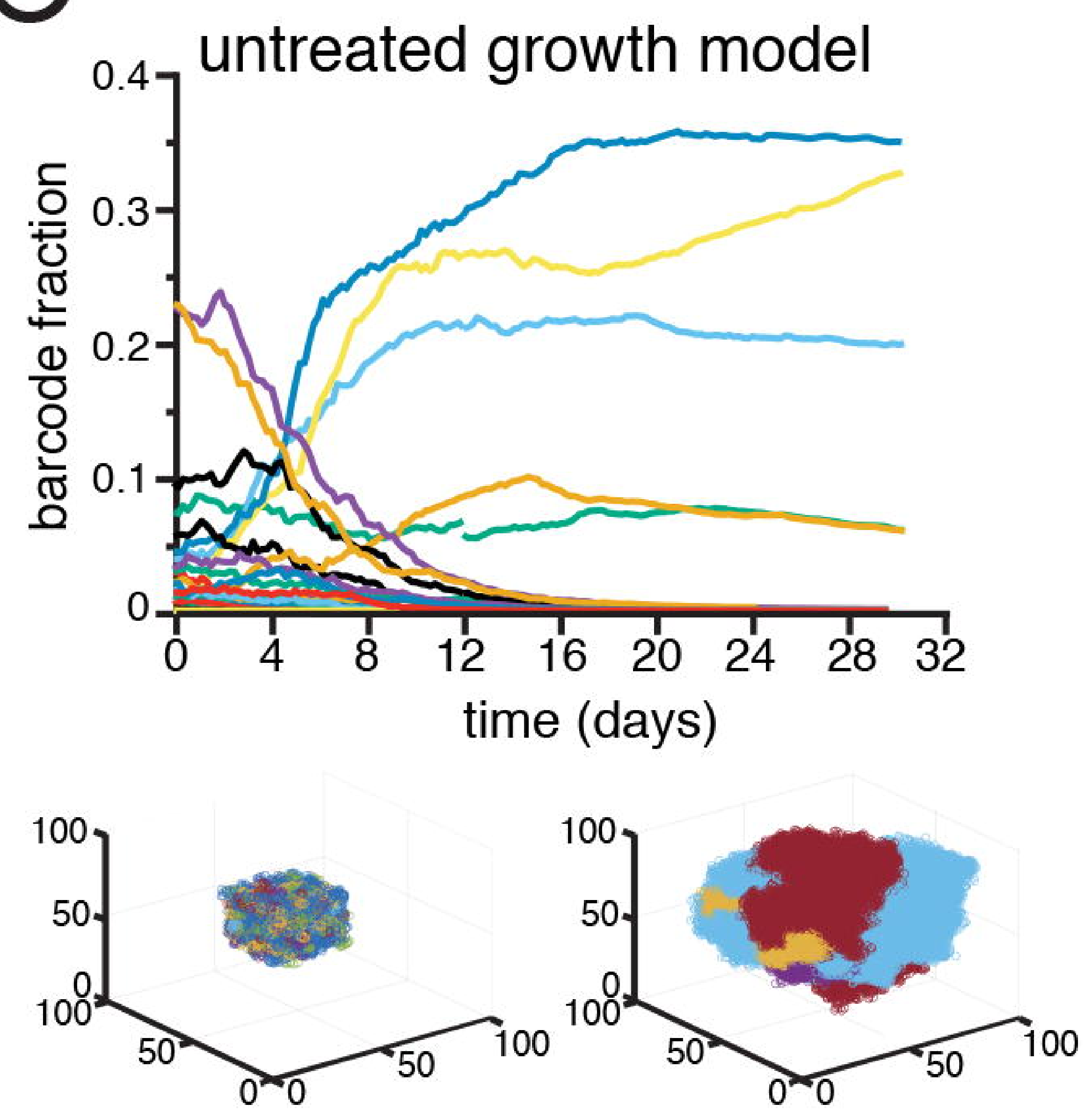

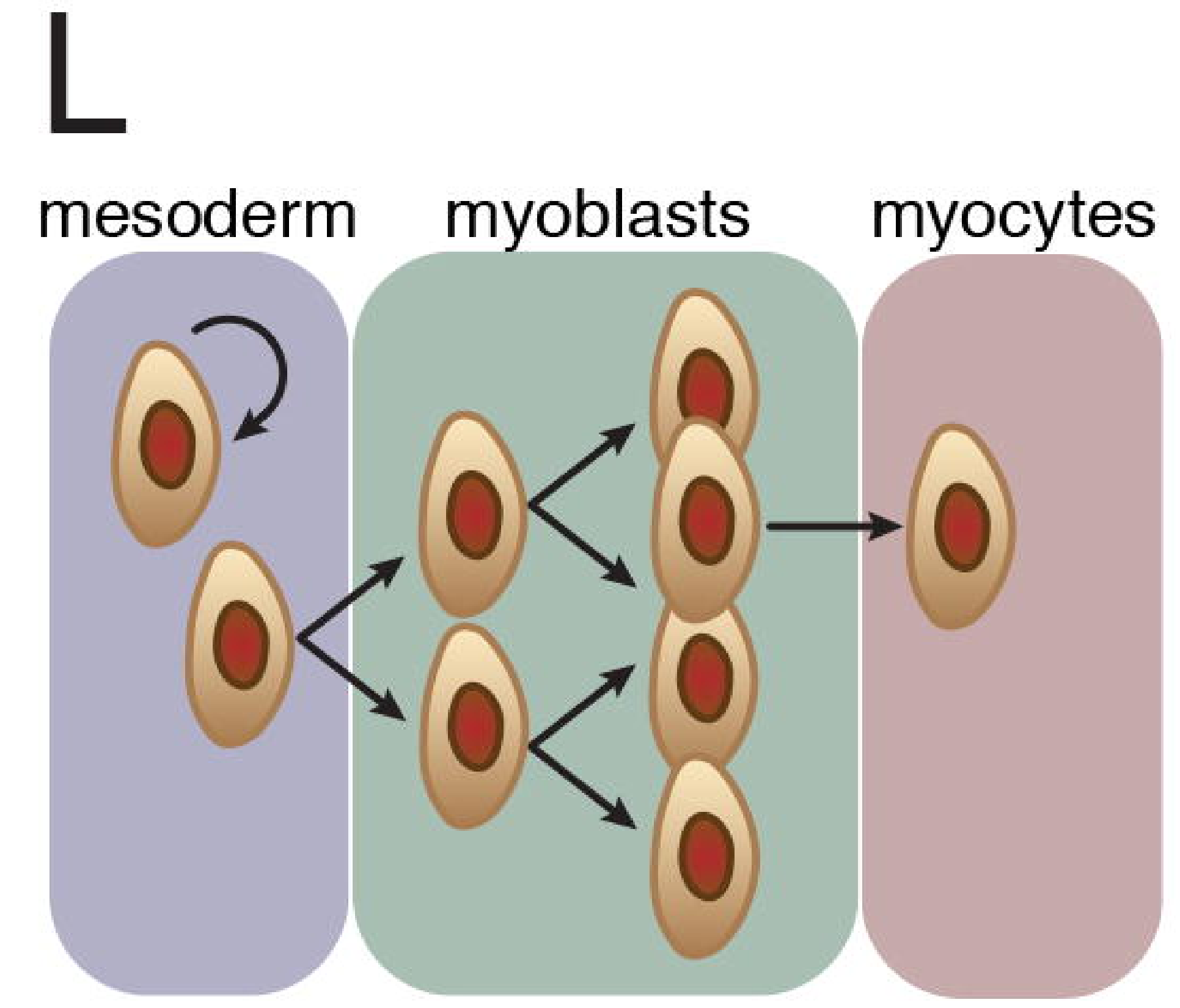

$\mathrm{P}$
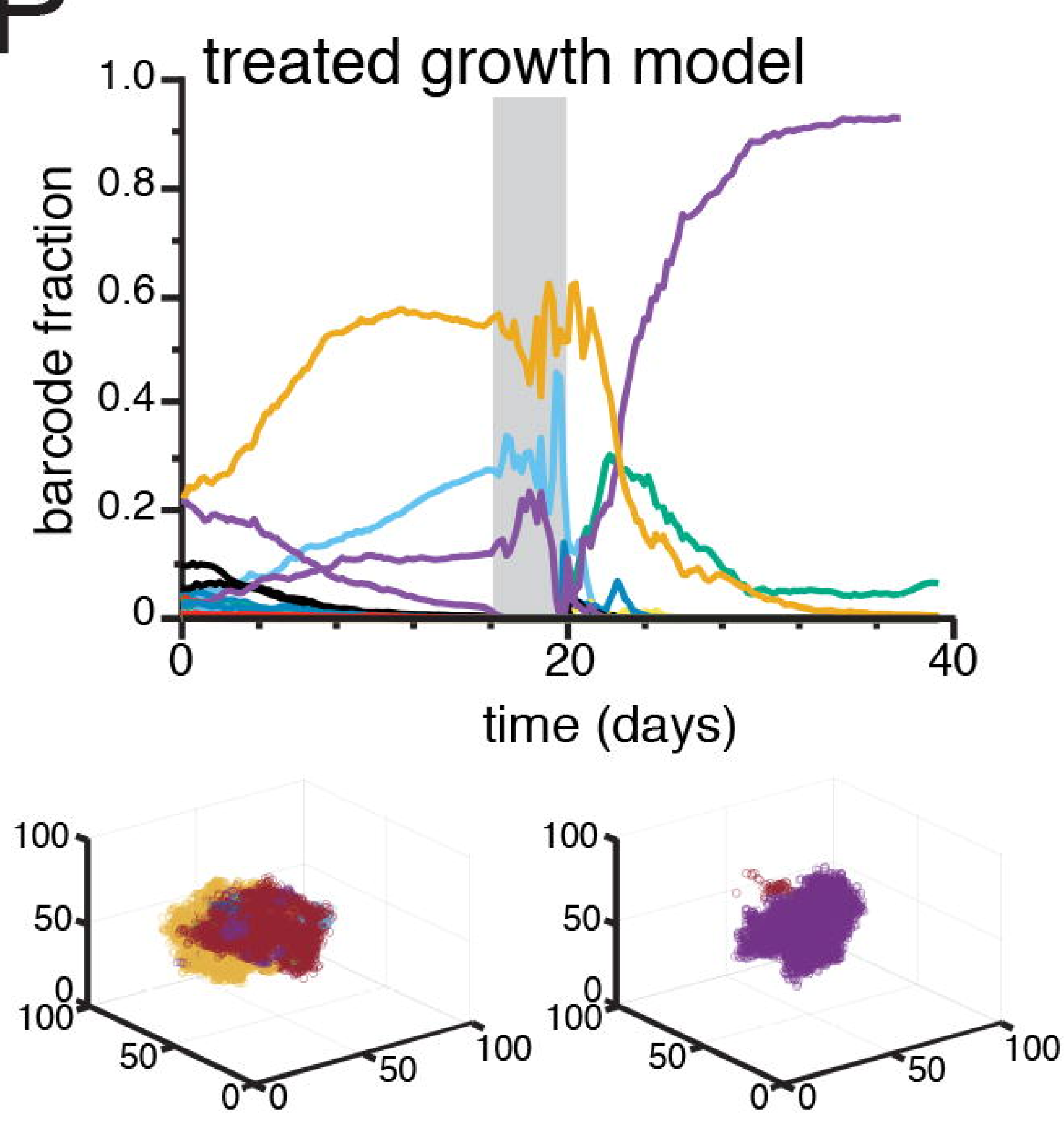
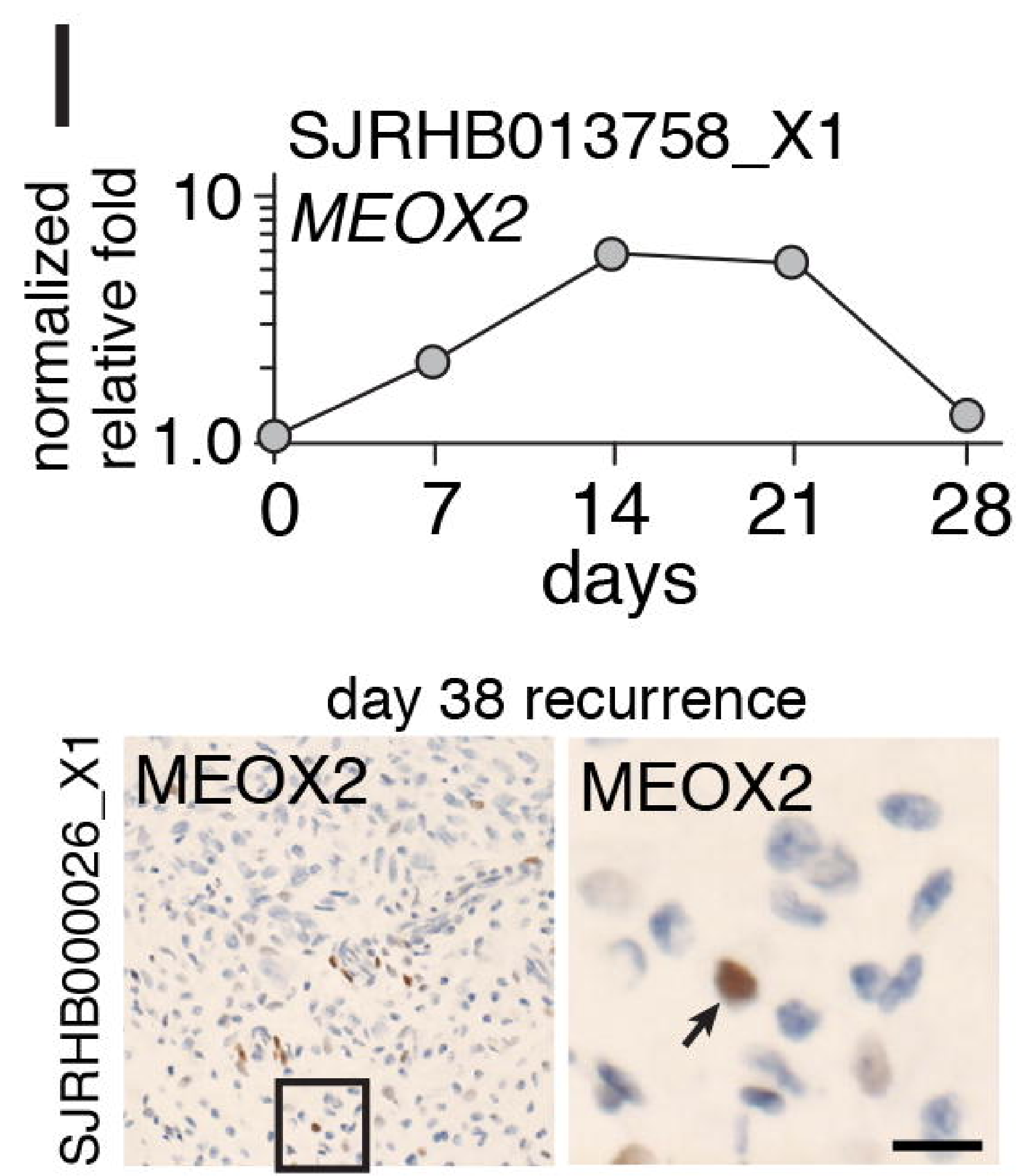

M untreated growth model

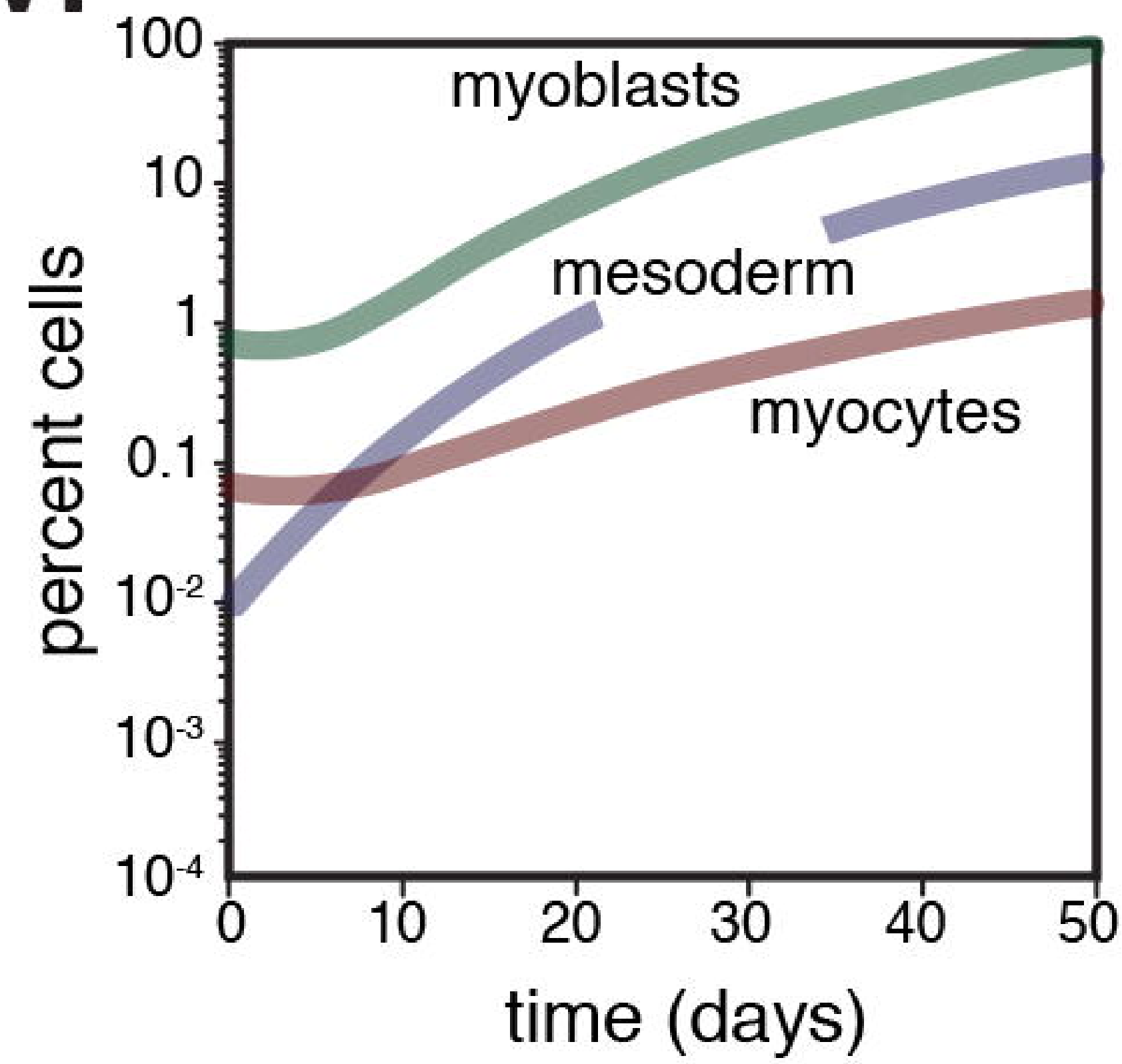

Q

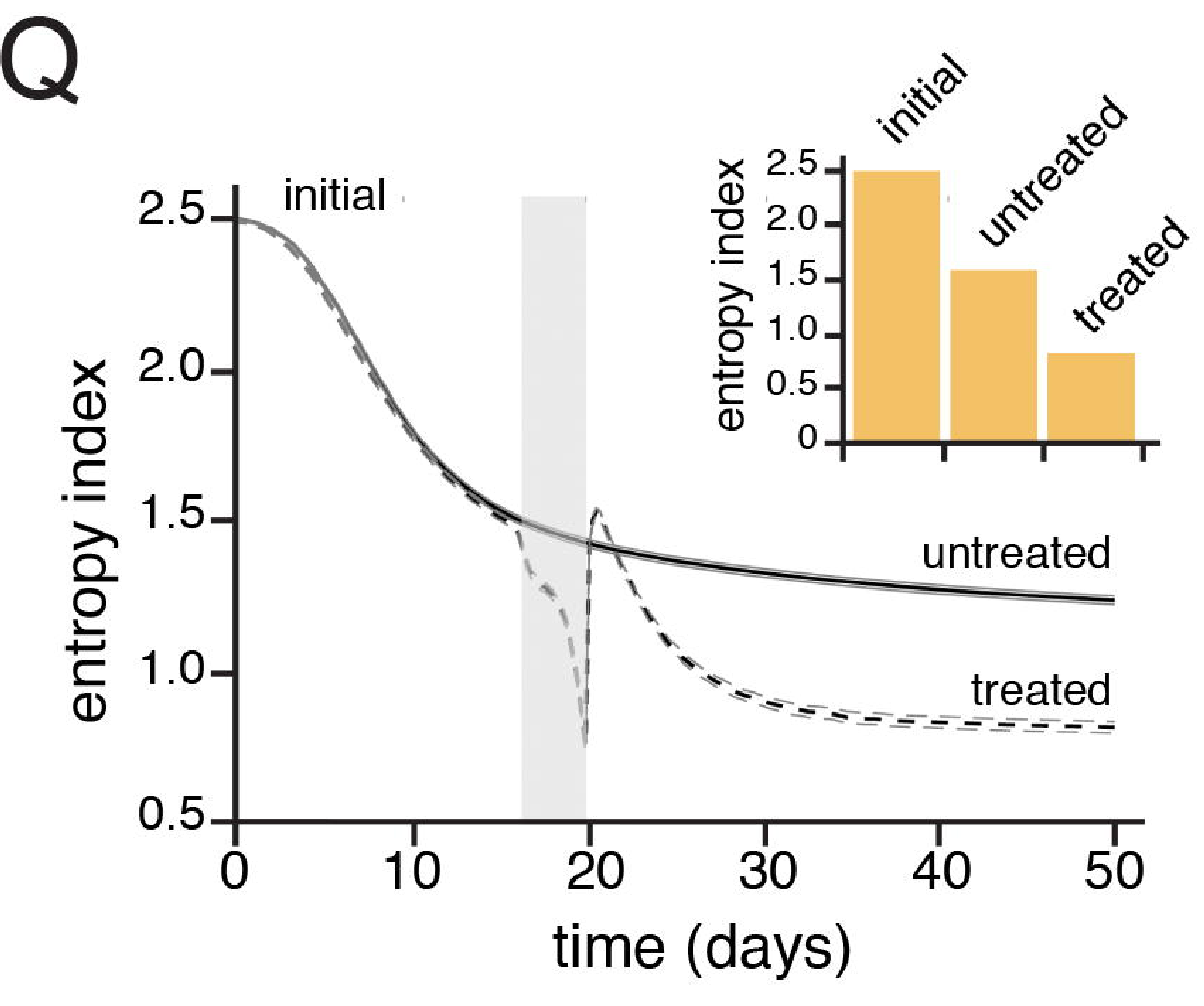


